\title{
An Efficient Macromodeling Methodology for Lateral Air Damping Effects
}

\author{
Yao-Joe Joseph Yang, Member, IEEE, and Po-Ching Yen
}

\begin{abstract}
In this paper, a macromodeling methodology for lateral air damping effect is presented. This methodology employs a simplified governing equation, the Quasi-3D (Q3D) Stokes equation, and an Arnoldi-based model-order-reduction algorithm. A finite-difference (FDM) solver based on the Q3D-Stokes equation is implemented, and then the Arnoldi-based algorithm is used to create macromodels from the system matrices generated by the solver. This methodology can also be realized by using commercial MEMS packages for solid-model generation, and by using commercial finite-element (FEM) thermal packages for system-matrix generation. The generated macromodels are compatible with system-level modeling simulators, such as SPICE, Saber, or Simulink for fast transient and frequency analyses. It is demonstrated that the macromodels are at least 600 times more efficient than the FDM Q3D Stokes solver, while are still capable of capturing the three-dimensional (3-D) effect that usually requires very expensive 3-D FEM Stokes-flow calculations. Experimental results of comb-drive devices show that the error of the macromodel is less than $10 \%$, which is a significant improvement when compared with the results by widely used 1-D analytical approaches. Finally, the guidelines of using this macromodeling methodology for typical MEMS devices are also provided. [1262]
\end{abstract}

Index Terms-Arnoldi algorithm, lateral air damping, macromodel, stokes equation, system-level analysis.

\section{INTRODUCTION}

A LARGE class of microelectromechanical systems (MEMS) devices, such as accelerometers, gyroscopes, switches, and micromirrors, must operate at significant air pressures. To understand the dynamic behaviors of these devices, the effects of the air surrounding their movable components can be critical [1]-[4]. It is well known that modeling three-dimensional (3-D) lateral air damping effects for MEMS devices requires considerable computational resources. For example, Fig. 1 shows a typical comb-drive device whose mechanism is widely used as the core sensing or actuating structures for MEMS devices [5]-[7]. For 3-D finite-element (FEM) or finite-difference (FDM) structural analysis of the device, the computational domains (meshes), which include the interdigital comb-structures and the folded-beams, are very complicated in terms of geometrical shapes. For 3-D FEM/FDM lateral-damping (fluidic) analysis of the device, the computational domains are even more complicated because they are in fact the air-films surrounding the structures. Because of this, 3-D FEM/FDM lateral-damping analysis for the comb

Manuscript received February 2, 2004; revised December 9, 2004. This work was supported by the National Science Council (Contract 92-2218-E-002-083), Taiwan, R.O.C. Subject Editor R. R. A. Syms.

The authors are with the Department of Mechanical Engineering, National Taiwan University, Taipei, Taiwan, ROC (e-mail: yjy@ntu.edu.tw).

Digital Object Identifier 10.1109/JMEMS.2005.845459

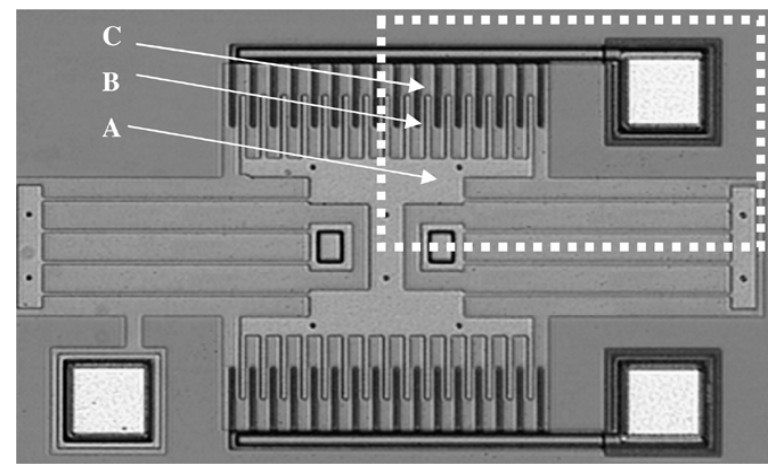

Fig. 1. The CCD picture of a comb-drive simulated and measured in this paper.

device not only requires intensive efforts on solid-modeling, but also requires significant computational resources even for steady incompressible-flow simulations. Therefore, most previous works on lateral air damping analysis were based on the 1-D analytical Stokes and Couette flow solutions [2], [4], [8]. The 1-D analytical approaches provide the first-order estimation of lateral air damping effects, and require almost zero computational resources so that they are intrinsically compatible with any system-level simulators. However, the 1-D approaches oversimplify the geometrical complexity of typical MEMS devices, and therefore the error is noticeable for most cases.

Recently, Aluru [9] developed a 3-D Stokes solver using the 3-D boundary-element method (BEM). The 3-D BEM approaches only need 2-D panels (meshes) on the surfaces of 3-D structures, so the required solid-modeling works are more straightforward than those of the 3-D FEM/FDM approaches. Furthermore, with certain numerical acceleration techniques, the computation efficiency of BEM solvers can be effectively increased [10], [11]. However, since the governing equations solved by the BEM techniques are in frequency domain, the simulated results are in fact for specific operating frequencies, and thus are not completely compatible with transient system-level analysis.

In this paper, we developed a methodology that employs a model-order-reduction (MOR) technique [12]-[16] to generate accurate time-domain macromodels from the 3-D FDM/FEM formulations of a Quasi-3D (Q3D) Stokes equation. The Q3D-Stokes equation is derived from the full 3-D Stokes equation by only considering the velocity component of interest. This simplified governing equation is in fact similar to the 1-D Stokes equation [2], while it is capable of capturing the important effects which the 1-D Stokes equation cannot provide. Another important advantage of this Q3D approach is that it 


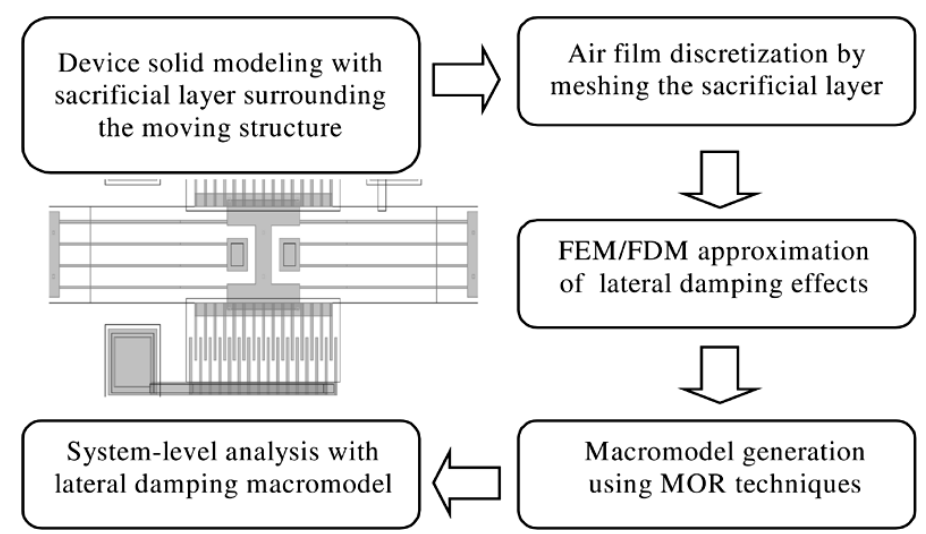

Fig. 2. Procedure of the efficient and accurate air damping modeling methodology for MEMS devices described in this paper.

TABLE I

GAS PROPERTIES USED IN THIS PAPER

\begin{tabular}{c|c|c}
\hline Viscosity coefficient & Gas density & Kinetic viscosity \\
\hline$\mu=18.5 \times 10^{-6} \mathrm{Ns} / \mathrm{m}^{2}$ & $\rho=1.1766 \mathrm{~kg} / \mathrm{m}^{3}$ & $\nu=15.73 \times 10^{-6} \mathrm{~m}^{2} / \mathrm{s}$ \\
\hline
\end{tabular}

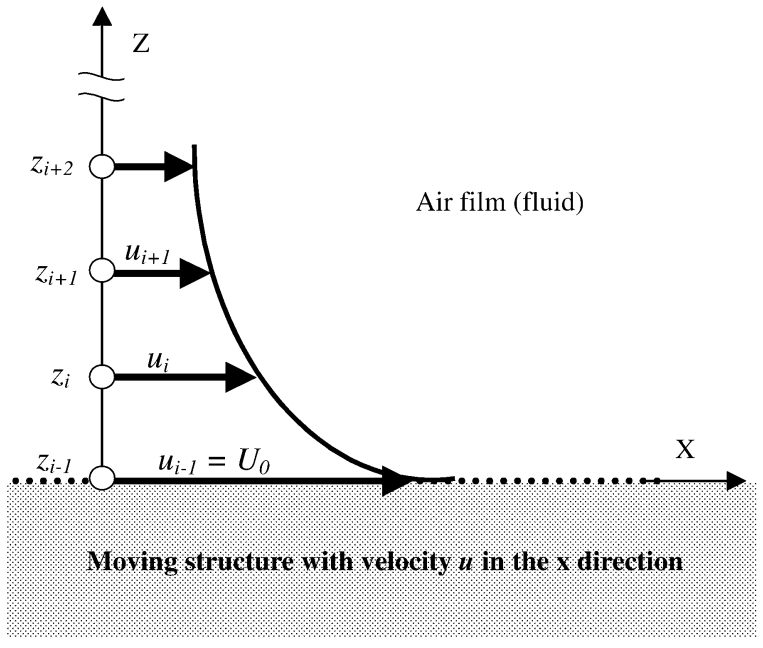

Fig. 3. Schematic of a lateral damping model for a 1-D case.

requires much less computational cost when compared with the approaches that directly solve the full 3-D Stokes equation.

The first step of this macromodeling methodology is to create 3-D solid models for the device to be modeled. This step is the same as the typical procedure of performing FEM//FDM fluidic simulations. However, we apply the special functionality of the commercially available MEMS modeling packages, such as Coventorware, IntelliSuite and MEMS-Pro [17]-[19], to $a u$ tomatically generate the 3-D solid model of the air films from 2-D mask layouts, by considering the air-films surrounding the structures as fictitious sacrificial layers. Then the FEM/FDM techniques are used to discretize the solid model. Since the governing equation is in time domain, the discretization step creates a system (set) of ordinary differential equations (ODEs) whose state variables are the velocity distribution in air films. Although the ODE system can be readily simulated using explicit or implicit integration techniques [20], [21], it might be so large that huge computational resources are required. Without direct time integration for the ODE system, the Arnoldi algorithm [14], [22] is applied to reduce the system of differential equations into a low-order system, the so-called macromodel [1]. The macromodels can be inserted into system-level simulators, such as SPICE, Saber, or Simulink, for transient and frequency-response analyses. Fig. 2 shows the flowchart of the proposed modeling procedure, which has been implemented as an automatic process in this paper.

This paper is organized as follows: the derivation of the governing equation for the fluidic damping effects is presented in Section II. The Arnoldi-based technique that will be used to generate the compact damping models is described in Section III. In Section IV, the simulated results are presented and discussed. Comparisons between the measured and simulated results are also demonstrated in this section. The discussions of appropriately using this macromodeling technique for MEMS devices are presented in Section V. The guidelines are also summarized. Finally, Section VI concludes this paper.

\section{Q3D STOKES EQUATION}

The complete governing equations for modeling lateral air damping is the Navier-Stokes equation and the continuity equation [23]

$$
\begin{aligned}
\rho \frac{D \vec{V}}{D t} & =-\nabla p+\mu \nabla^{2} \vec{V} \\
\nabla \cdot \vec{V} & =0
\end{aligned}
$$

where $p$ is the pressure, $\vec{V}=\left[\begin{array}{lll}u & v & w\end{array}\right]^{T}$ is the velocity vector, $\mu$ is the viscosity of the air, and $u, v$, and $w$ are the velocity components in $x, y$, and $z$ direction, respectively. Since the air is assumed to be incompressible, the density $\rho$ is constant. Note that in our model, the gas rarefaction effect is not considered. More discussion on the modeling error caused by the ignorance of the gas rarefaction effect will be provided in Section V. 


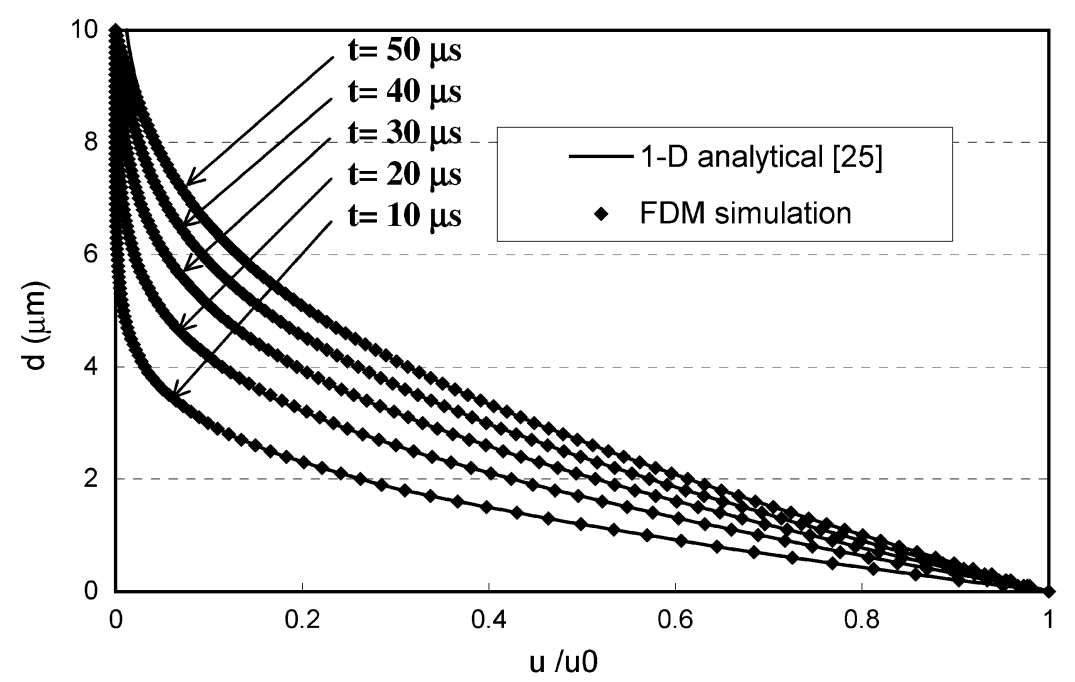

(a)

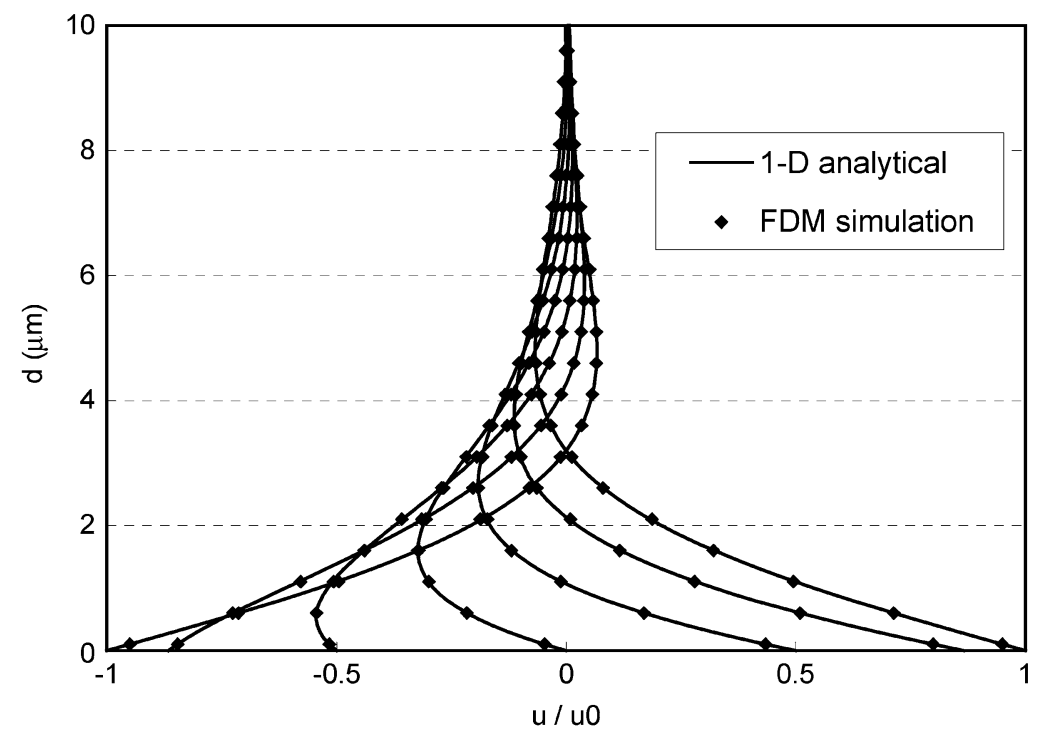

(b)

Fig. 4. Velocity profiles of the analytical solution and FDM solver for a plate (a) moving at constant velocity. (b) Oscillating at $1 \mathrm{MHz}$.

For our case, the imposed pressure gradient is assumed to be zero, so the first term on the right-hand side of (1) is eliminated. The governing equation is further simplified by neglecting the effects of the velocity components which are perpendicular to the direction of the prescribed in-plane motion. In other words, if the imposed velocity of the moving structure is in $x$ direction, $u$ is assumed to be much larger than $v$ and $w$. Consequently, the continuity equation becomes trivial and is not considered. Then (1) becomes

$$
\frac{\partial u}{\partial t}+(u \cdot \operatorname{grad}) u=v \nabla^{2} u
$$

where $v=\mu / \rho$ is the kinetic viscosity.

Note that these simplifications (i.e., neglect $v, w$ and the continuity equation) are actually based on the same assumptions for deriving the 1-D Stokes equation [23]. Also note that this simplification gives rise to the modeling error due to neglecting the "end effect." Extensive discussions on this will be provided in Section IV.
Assume that the moving structure is in oscillation with an angular frequency $\omega$. Let $a_{0}$ be the amplitude of oscillation, $u_{0}\left(=a_{0} \omega\right)$ be the amplitude of the fluid velocity, and $L$ be the characteristic length of the moving structure (e.g., its characteristic dimension). The nonlinear term in (3) is of the order

$$
(u \cdot \operatorname{grad}) u \sim \frac{u_{0}^{2}}{L} \sim \frac{a_{0}^{2} \omega^{2}}{L}
$$

and the time dependent term of (3) will be of the order

$$
\frac{\partial u}{\partial t} \sim u_{0} \omega \sim a_{0} \omega^{2} .
$$

Since typically the amplitude of the oscillation $\left(a_{0}\right)$ is much smaller than the dimension of the moving structure $(L)$, the nonlinear term in (3) is negligible. Finally, the simplified equation, which we call the Q3D Stokes equation, is

$$
\frac{\partial u}{\partial t}=v \nabla^{2} u=v\left(\frac{\partial^{2} u}{\partial x^{2}}+\frac{\partial^{2} u}{\partial y^{2}}+\frac{\partial^{2} u}{\partial z^{2}}\right) .
$$




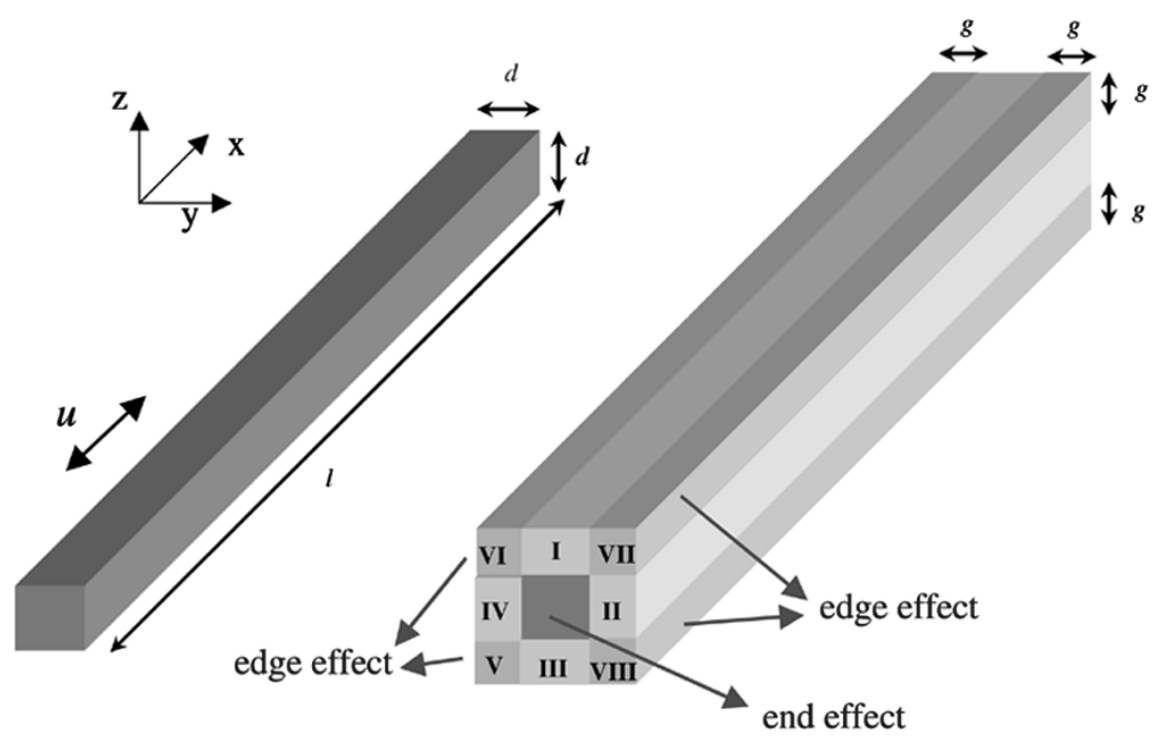

(a)

(b)

Fig. 5. The schematic for illustrating the edge effect and the end effect of a comb drive finger. (a) A single finger structure moving with velocity $u$. (b) Air Volumes surrounding the finger structure.

Obviously, the Q3D-Stokes equation is very similar to the 1-D Stokes equation [2], [4], except that the effects of $u$ 's variations in $y$ and $z$ directions will also be considered in the Q3D-Stokes equation. Also, (6) is in fact in the same form of the 3-D energy equation (heat transfer diffusion equation), which implies that simulating the Q3D-Stokes equation can be easily achieved by using the diffusion equation solvers provided by most commercial FEM packages.

In this paper, based on the Q3D-Stokes equation, a transient FDM solver is developed for modeling MEMS lateral damping effect. In the following sections, we will demonstrate that this FDM Q3D-Stokes solver not only can simulate transient lateral damping effects, but also can be used to generate macromodels for ultraefficient system-level dynamical modeling. With limited computational resources, these capabilities usually cannot be carried out by typical commercial computational fluidic dynamics (CFD) solvers for modeling the devices with very complicated geometries.

\section{ARnOldi-BASEd Model ORder Reduction TechniQue}

Although the FDM Q3D-Stokes solver is ready for transient analysis, it still requires large computational resources for very complicated structures (e.g., the comb-drive device shown in Fig. 1). Fortunately, since the governing equation, the Q3DStokes equation (6), is a linear equation, the system matrices generated by the FDM approximation process can be reduced by the Arnoldi-based MOR technique, which will be described as follows.

The dynamic system equations formulated by the FDM discretization of (6) can be written as

$$
\begin{aligned}
& \dot{\vec{u}}=\mathbf{A} \cdot \vec{u}+\mathbf{B} \cdot v_{\text {in }} \\
& \vec{y}=\mathbf{C}^{T} \cdot \vec{u}+\mathbf{D} \cdot v_{\text {in }}
\end{aligned}
$$

where $\mathbf{A}$ is an $n$ by $n$ matrix and $n$ is the total number of nodes, $\vec{u}$ is the vector which contains the unknown velocity distribution on each node, the input function $v_{\text {in }}$ is the imposed velocity on the moving boundary of the computational domain, and the modal shape of the moving boundary is presented in $\mathbf{B}$. In this case, we carefully formulate $\mathbf{C}$ and $\mathbf{D}$, so that the output $\vec{y}$ will be the frictional shear (calculated by Newtonian law of viscosity) on the plate. Detailed description on how these system matrices are generated is presented in Appendix I.

In Laplace domain, the transfer function of the system is

$$
T(s)=\mathbf{C}^{T}(\mathbf{I} s-\mathbf{A})^{-1} \mathbf{B}+\mathbf{D}=\mathbf{C}^{T}\left(\mathbf{I}-s \mathbf{A}^{-1}\right)^{-1} \vec{b}+\mathbf{D}
$$

where

$$
\vec{b}=-\mathbf{A}^{-1} \mathbf{B}
$$

After expanding the transfer function in Taylor series about $s=$ 0 , we obtain

$$
\begin{aligned}
T(s) & =\mathbf{C}^{T}\left(\mathbf{I}+s \mathbf{A}^{-1}+s^{2} \mathbf{A}^{-2}+\cdots\right) \vec{b}+\mathbf{D} \\
& =\sum_{k=0}^{\infty} m_{k} s^{k}+\mathbf{D}
\end{aligned}
$$

where $m_{k}$ are the coefficients of the Taylor series

$$
m_{k}=\mathbf{C}^{T}\left(\mathbf{A}^{-k}\right) \vec{b} .
$$

The Taylor expansion can be truncated to approximate the transfer function $T(s)$. Since $\mathbf{A}^{-k} \vec{b}$ quickly line up with a single eigenvector, this moment matching procedure is usually numerically unstable. Therefore, we apply the Arnoldi-based algorithm to stably compute orthogonal bases $\vec{v}_{i}$ that span the Krylov subspace

$K_{q}\left(\mathbf{A}^{-1}, \vec{b}\right)=\operatorname{span}\left\{\vec{b}, \mathbf{A}^{-1} \vec{b}, \mathbf{A}^{-2} \vec{b}, \ldots, \mathbf{A}^{-(q-1)} \vec{b}\right\}$. 
(10)

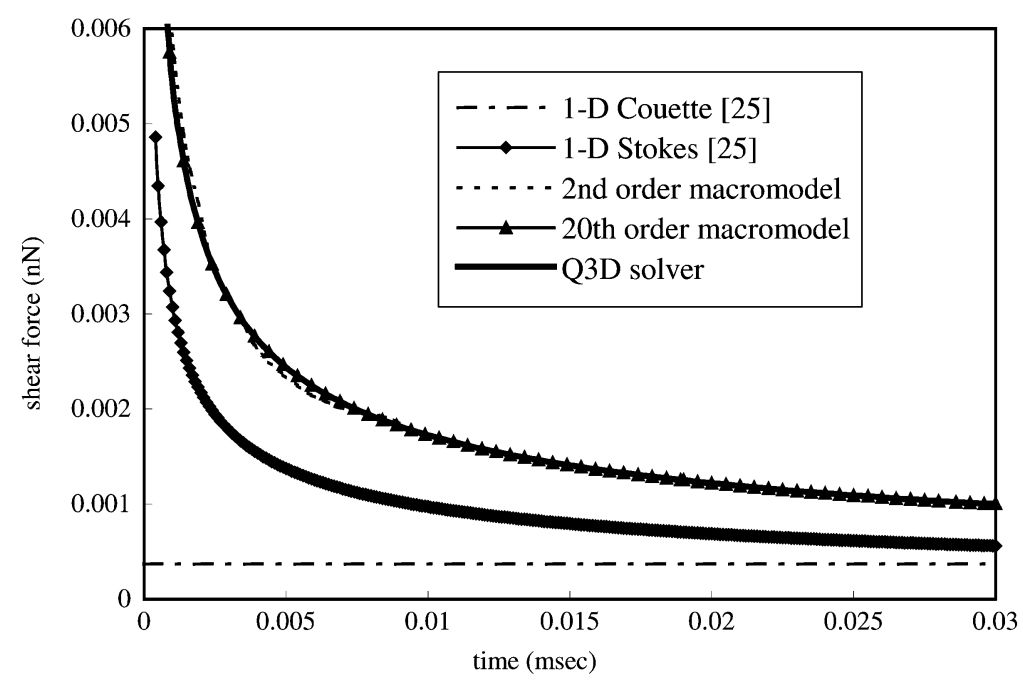

(a)

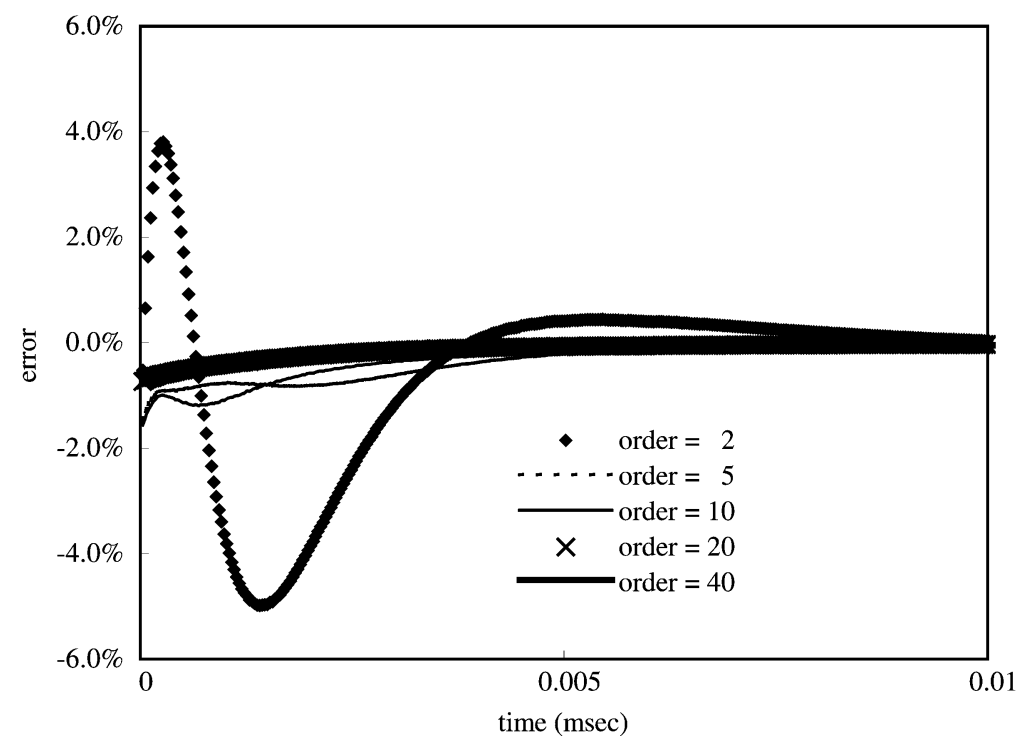

(b)

Fig. 6. The results of transient simulations of the FDM Q3D-Stokes solver, the 1-D analytical solution, and the Q3D-Stokes macromodels. The simulated velocity of the moving comb structure is $1000 \mu \mathrm{m} / \mathrm{s}$.

Given the matrix $\mathbf{V}_{\mathbf{q}}$ whose columns are $\vec{v}_{i}$, the Arnoldi algorithm reduces the system matrices into:

$$
\mathbf{V}_{\mathbf{q}}^{\mathbf{T}} \mathbf{A V}_{\mathbf{q}}=\mathbf{A}_{\mathbf{q}}, \quad \mathbf{V}_{\mathbf{q}}^{\mathbf{T}} \mathbf{B}=\mathbf{B}_{\mathbf{q}}, \quad \mathbf{V}_{\mathbf{q}}^{\mathbf{T}} \mathbf{C}=\mathbf{C}_{\mathbf{q}}
$$

Finally, (9) can also be reduced as

$$
T_{q}(s)=\mathbf{C}^{T} \mathbf{V}_{\mathbf{q}}\left(\mathbf{I}_{\mathbf{q}}-s \mathbf{A}_{\mathbf{q}}\right)^{-1} \mathbf{V}_{\mathbf{q}}^{T} \vec{b}+\mathbf{D}
$$

and the corresponding time domain reduced-order macromodel is

$$
\begin{aligned}
\dot{\vec{u}} & =\mathbf{A}_{\mathbf{q}} \cdot \vec{u}+\mathbf{B}_{\mathbf{q}} \cdot v_{\text {in }} \\
\vec{y} & =\mathbf{C}_{\mathbf{q}}^{T} \cdot \vec{u}+\mathbf{D} \cdot v_{\text {in }} .
\end{aligned}
$$

Note that the reduced system, as shown in (12), has the same input $\left(v_{\text {in }}\right)$ and output $(\vec{y})$ as those in (7). Since the typical rank of the reduced system is much smaller than that of the original system, its computational efficiency for simulating transient responses and frequency responses is much higher. This reduced system is the so-called macromodel which can be used for system-level transient or frequency analyzes.

\section{Simulated Results}

Fig. 1 shows the picture of a simulated and measured combdrive structure fabricated by the MUMPs ${ }^{\circledR}$ process [24]. In terms of the geometrical characteristics, we classify the computational domains (air films) as three types. The first type is the air film above or below the plate-type structure. An example of this is shown at Point A in Fig. 1. At this location, the 1-D analytical solutions for lateral damping effects are reasonably accurate because this area can be approximated as a big flat plate. Point B and Point $\mathrm{C}$ indicate the typical locations of the second type and 


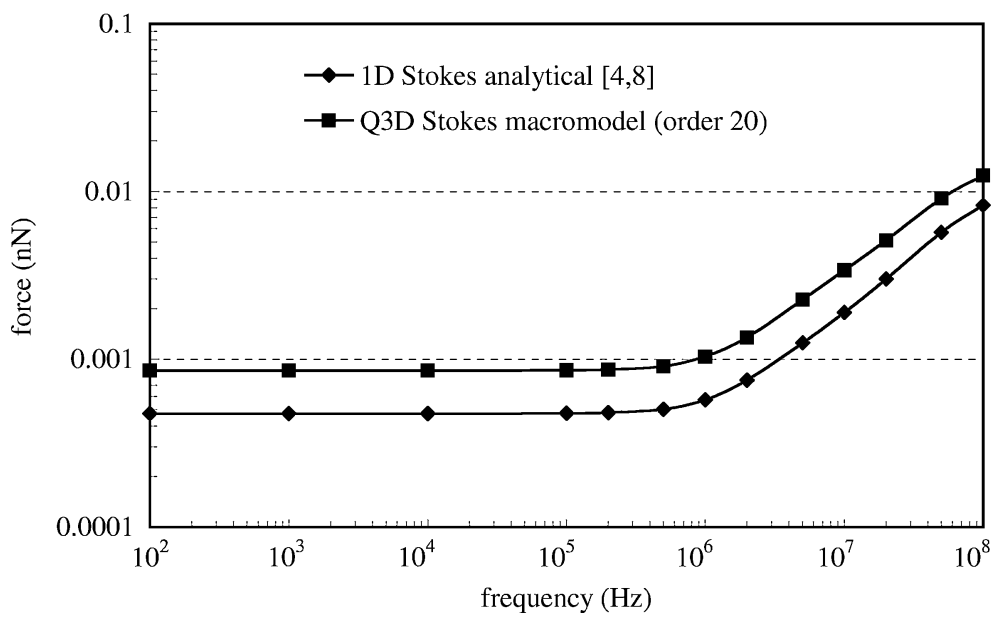

(a)

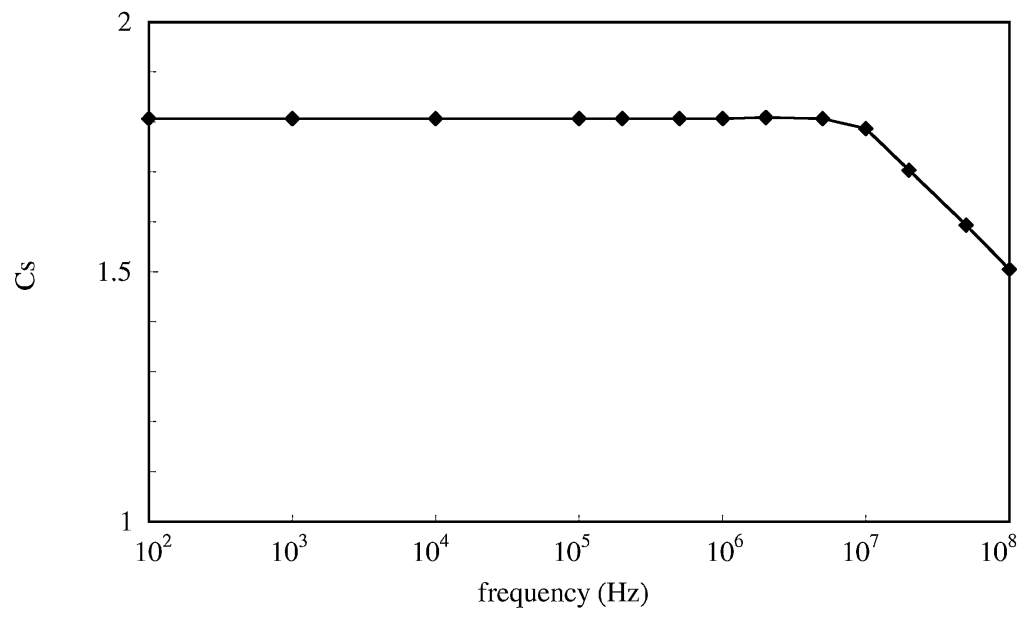

(b)

Fig. 7. (a) Frequency responses for the structure shown in Fig. 5. (b) Ratio between the Q3D-Stokes result and the 1-D result versus frequency.

the third type, respectively. At these locations, the 1-D analytical solutions are not accurate any more because the edge effect (Point B) and the end effect (Point C) will need to be considered due to the complexity of the geometry. In the following subsections, we will discuss both the edge effect and the end effect in detail.

Because of the geometric symmetry of the comb structure, the computational domain of this case consists of the air films that surrounds one quarter of the device, as indicated by the dashed lines in Fig. 1. The air properties are listed in Table I. The total number of the nodes used for discretizing the air films is 626 745 . Note that this node number is determined by a mesh convergence test which shows that the simulated damping forces converge as the number of nodes is equal to or greater than this number. More discussions regarding to the study of mesh convergence under different conditions will be provided in subsequent sections.

It has to be pointed out that due to the complexity of the geometry of the interdigital structure, if using the FEM/FDM approaches, it is quite typical that more than hundred thousands of nodes are required to discretize the air films surrounding the structure. Therefore, solving this complicated case using commercial FEM CFD packages, which simultaneously solve the full Navier-Stokes equation and the continuity equation in transient, is usually impossible because huge computational resources are required. However, since our approach uses the simplified governing equation, the Q3D-Stokes equation, even transient calculations are feasible for very complicated structure.

\section{A. Physical Characteristics of the Q3D-Stokes Solver}

1) Comparison With 1-D Analytical Solutions: At Point A in Fig. 1, the top or the bottom surface of structure can be approximated as a semiinfinite plate. The schematic is shown in Fig. 3. Assuming that the plate is initially at rest and acquires a velocity of $u_{0}$ at $t=0$, the 1-D analytical solution of velocity profile is [25]

$$
u=u_{0}\left[1-\operatorname{erf}\left(\frac{z}{2 \sqrt{v t}}\right)\right]
$$

where $z$ is the distance from the moving boundary, $u_{0}$ is the plate velocity, and erf is the error function. The simulated results by the FDM Q3D-Stokes solver and the analytical solutions match each other very well, as shown in Fig. 4(a). The curves in the figure are the velocity profiles for different time steps. 


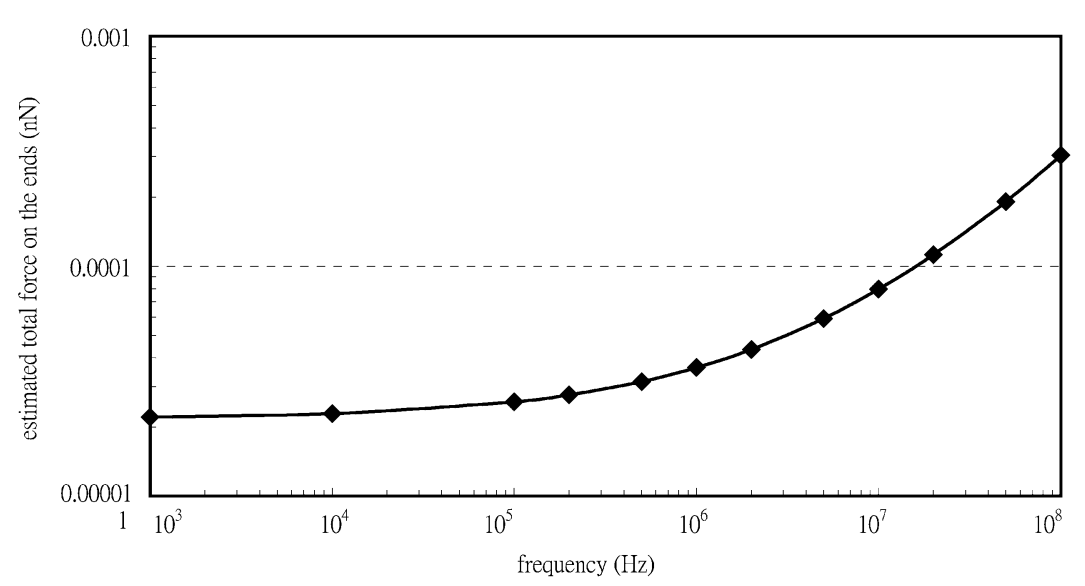

(a)

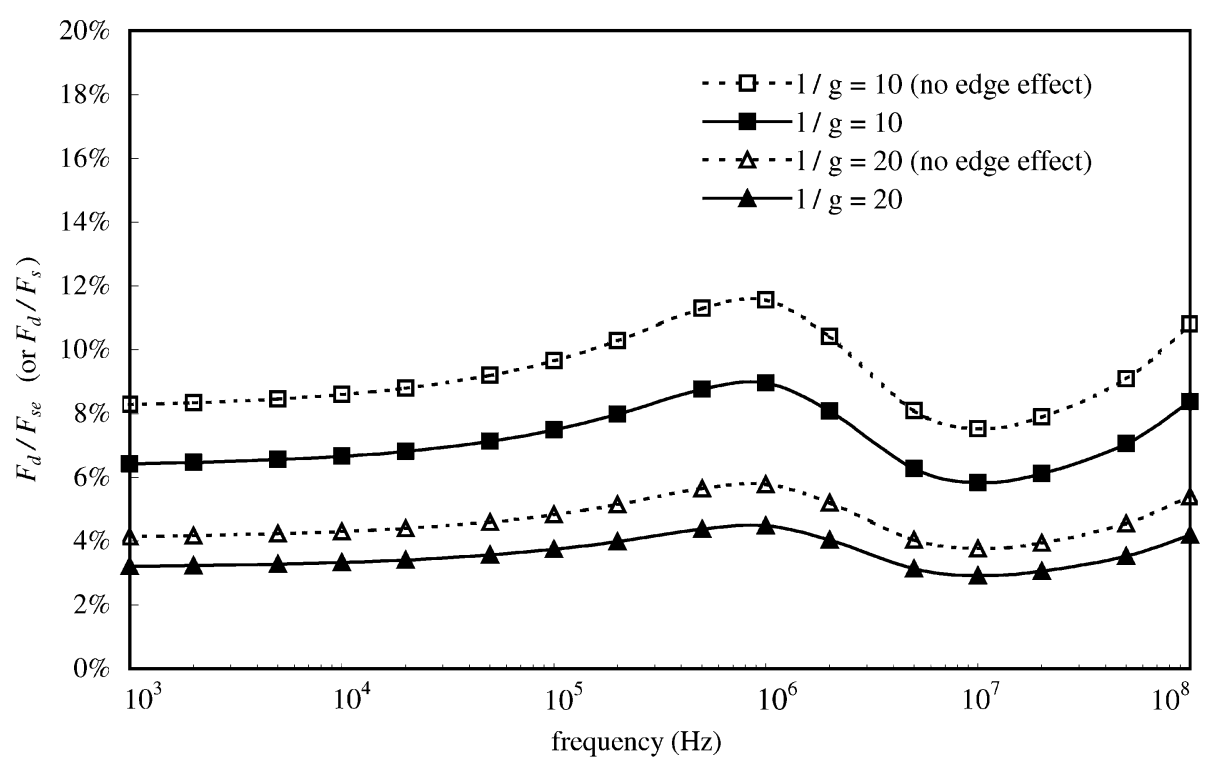

(b)

Fig. 8. (a) Total shear force and the total drag force (end effect) for the beam shown in Fig. 5 ( $l=20 \mu \mathrm{m}, d=g=2 \mu \mathrm{m}$ ). (b) $F_{d} / F_{\text {se }}$ versus frequency for different aspect of the beam $(d=g=2 \mu \mathrm{m})$.

For the case that a moving surface oscillating in periodic motions, the corresponding 1-D solution of the velocity profiles of the air film is

$$
u=u_{0} \cos \left(\omega t-\sqrt{\frac{\omega}{2 v}} z\right) e^{-\sqrt{\frac{\omega}{2 v}} z}
$$

where $\omega$ is the oscillating angular frequency. Fig. 4(b) shows the velocity profiles estimated by the 1-D analytical solution and those calculated by the FDM Q3D-Stokes solver (1 MHz oscillation frequency). The curves shown in the figure are the velocity profiles for $\pi / 6$ intervals in a half oscillation period after reaching sinusoidal steady-state. Again, the FDM results and the analytical results are in good agreement. Fig. 4 demonstrates that the FDM Q3D-Stokes solver can accurately calculate the fluidic behaviors for the region where the assumptions of the 1-D analytical solutions apply.

2) Edge Effect: At Point B in Fig. 1, the structure is in fingershape. The velocity profiles in the air films around the finger are no longer like those near the center of a flat plate, and the edge effect takes place. In order to illustrate the edge effect, we

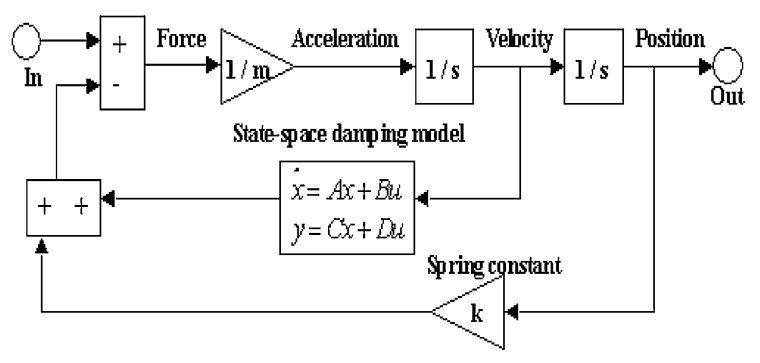

Fig. 9. A Simulink system level schematic with a lateral damping macromodel.

divide the air films surrounding a single finger into Air Volumes $I-V I I I$, as shown in Fig. 5. If using the 1-D analytical solutions (as described previously), the lateral damping force acting on each of the four surfaces adjacent to Volumes I, II, III, and IV is estimated by

$$
F_{\text {damping }}=\tau \cdot A=\frac{d u}{d z} \cdot \mu \cdot A
$$

where $\tau$ is the shear stress acting on a surface with area $A$. 
TABLE II

Comparison of Simulation Times Between the FDM Q3D-STOKES SOlVER AND THE MACROMODELS

\begin{tabular}{|c|c|c|c|c|}
\hline \multicolumn{5}{|c|}{ Results by the FDM Q3D-Stokes Solver (full model) } \\
\hline Simulation time $(=F)$ & \multicolumn{4}{|c|}{$63.95 \mathrm{sec}$} \\
\hline \multicolumn{5}{|c|}{ Results by the Macromodel (reduced models) } \\
\hline Types & $2^{\text {nd }}$ order & $5^{\text {th }}$ order & $10^{\text {th }}$ order & $20^{\text {th }}$ order \\
\hline Generation time & $12.17 \mathrm{sec}$ & $25.42 \mathrm{sec}$ & $45.02 \mathrm{sec}$ & $85.11 \mathrm{sec}$ \\
\hline Simulation time $(=\mathbf{R})$ & $0.013 \mathrm{sec}$ & $0.025 \mathrm{sec}$ & $0.051 \mathrm{sec}$ & $0.103 \mathrm{sec}$ \\
\hline Speed-up factor $(=F / R)$ & 4920 & 2558 & 1294 & 621 \\
\hline
\end{tabular}

TABLE III

Dimensions of Six Tested Comb-Drive DeVices With DifFerent Folded BeAm LenGthS

\begin{tabular}{c|c|c|c|c|c|c}
\hline & Device 1 & Device 2 & Device 3 & Device 4 & Device 5 & Device 6 \\
\hline beam width $(\boldsymbol{\mu m})$ & 2 & 2 & 2 & 2 & 2 & 2 \\
\hline beam length $(\boldsymbol{\mu m})$ & 168 & 180 & 192 & 204 & 216 & 228 \\
\hline structure thickness $(\boldsymbol{\mu m})$ & 2 & 2 & 2 & 2 & 2 & 2 \\
\hline finger width $(\boldsymbol{\mu m})$ & 3 & 3 & 3 & 3 & 3 & 3 \\
\hline finger length $(\boldsymbol{\mu m})$ & 40 & 40 & 40 & 40 & 40 & 40 \\
\hline finger gap $(\boldsymbol{\mu m})$ & 3 & 3 & 3 & 3 & 3 & 3 \\
\hline finger overlap $(\boldsymbol{\mu m})$ & 20 & 20 & 20 & 20 & 20 & 20 \\
\hline comb-to-substrate gap $(\boldsymbol{\mu m})$ & 2 & 2 & 2 & 2 & 2 & 2 \\
\hline effective mass $(\boldsymbol{\mu g})$ & 0.04295 & 0.04328 & 0.04362 & 0.04395 & 0.04428 & 0.04461 \\
\hline effective spring constant $(\boldsymbol{\mu} \mathbf{N} / \boldsymbol{\mu m})$ & 0.6448 & 0.5414 & 0.4519 & 0.3698 & 0.3168 & 0.2664 \\
\hline measured resonant frequency $\mathbf{( H z )}$ & 19500 & 17800 & 16200 & 14600 & 13400 & 12300 \\
\hline
\end{tabular}

Since the assumption of semiinfinite plate is no longer applicable on the four narrow surfaces that are adjacent to Air Volumes I, II, III, and IV, the error of the 1-D analytical solutions might be considerable. One of the major contributions of the error comes from the fact that the 1-D approaches neglect the damping effects from Volumes V, VI, VII, and VIII, which we call the edge effect. For example, when the finger is moving, the velocity profiles in Volume I and Volume II will also be affected by the fluid in Volume V, which will introduce additional damping that the 1-D analytical solutions ignore completely. Since the FDM Q3D-Stokes solver discretizes the whole air films surrounding the comb drive structure, it will properly take into consideration the edge effects of the complex interdigital geometry. Fig. 6(a) shows the transient simulated results by the FDM Q3D-Stokes solver and the 1-D analytical solution [25] as the finger-type structure (Fig. 5) moves with step velocity of $1000 \mu \mathrm{m} / \mathrm{s}$ at $t=0$ (initially at rest). In this case, the finger length $l$ and the finger width $d$ are 20 and $2 \mu \mathrm{m}$, respectively. The gap $g$ is $2 \mu \mathrm{m}$. The total number of nodes for meshing the air file of the finger-type structure is 84442 . The transient responses (the total viscous force) reach steady-state values at about $0.3 \mathrm{~ms}$. The results indicate that the 1-D analytical models underestimate the damping forces because of neglecting the edge effect.

Fig. 7(a) shows the frequency response of the Q3D macromodel (order 20) for the finger structure shown in Fig. 5. The figure also presents the result by the 1-D analytical model (1-D Stokes with continuum boundary condition) [4], [8], which is shown in (16)

$$
F_{s}=\frac{4 \mu l d U_{0} q}{\tanh (q g)}
$$

where $q=\sqrt{j \omega / v}$ is a complex frequency variable. The shear force estimated by the 1-D model is always smaller than that estimated by the Q3D-Stokes model because the 1-D model does not account for the edge effect. The parameter $c_{s}$, which is defined as the ratio between the Q3D-Stokes result and the 1-D result, is shown in Fig. 7(b). Note that $c_{s}$ can be considered as the compensation factor of the edge effect for the finger-type structure shown in Fig. 5. Also, it is predicable that $c_{s}$ will approach to 1 for large thin-plate structures because the edge effect becomes insignificant for those structures.

3) End Effects: As discussed in Section II, the motivation of using the Q3D-Stokes equation is to avoid very expensive computations for solving the full 3-D Stokes equation, while still to provide more accurate results than widely used 1-D analytical approaches. Although the FDM Q3D-Stokes solver can effectively model the edge effect that is completely ignored by the 1-D approaches, it is not capable of accounting for the drag force against the end (tip) of each finger (Point $\mathrm{C}$ in Fig. 1), which we call the end effect. More precisely, the end effect is defined as the drag force against the surface whose normal is aligned with the direction of motion. Here, we will illustrate the influences of ignoring the end effects for modeling comb fingers. Note that the analysis described here is also applicable to estimate the errors of using the 1-D analytical solutions [2], [4], [8] to model lateral damping effect.

Again, we use the structure shown in Fig. 5. Here we use the 1-D analytical solution [4], [8] to estimate the total shear force on the four side-surfaces of the finger

$$
F_{\text {se }}=\frac{4 c_{s} \mu l d U_{0 q}}{\tanh (q g)}
$$




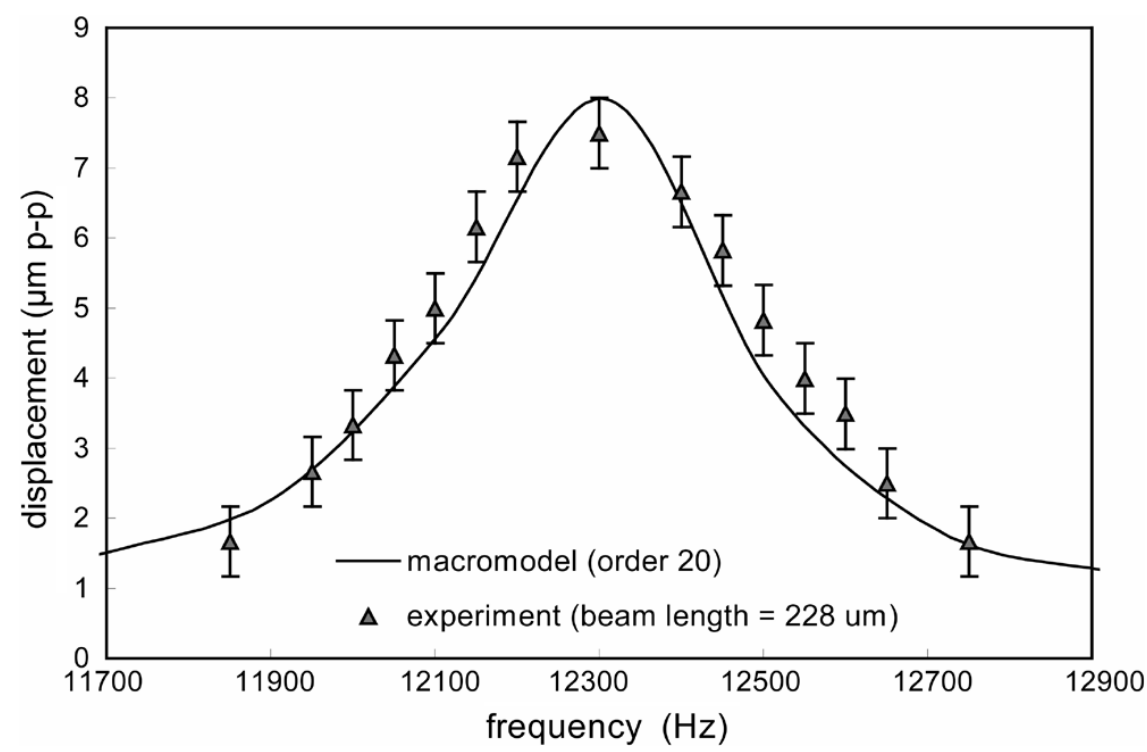

Fig. 10. Frequency response of the twentieth-order macromodel of the comb drive device. The length of the folded beam is $228 \mu \mathrm{m}$.

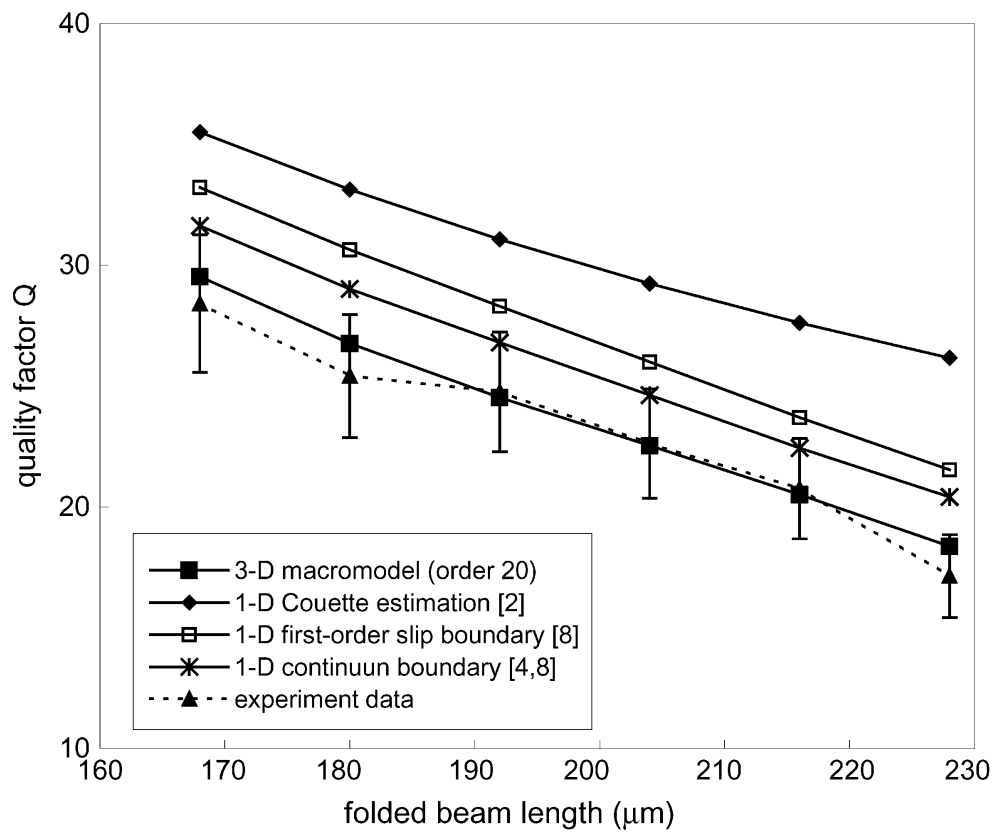

Fig. 11. Simulated and measured comb-drive quality factors versus different folded-beam lengths.

where the variable $c_{s}$, which is a frequency-dependent parameter as shown in Fig. 7(b), is the compensation factor for the edge effect.

For the end effect, we use the model of an oscillating sphere [26] to approximately estimate total drag force against both ends of the beam. The total drag force on a sphere oscillating at a velocity of $U_{0} \cos (\omega t)$ is

$$
F_{d}=M_{d} U_{0} \omega\left(k \sin (\omega t)-k^{\prime} \cos (\omega t)\right)
$$

where $M_{d}=\left(4 \pi r^{3} / 3\right), k=(1 / 2)+(9 / 4) \sqrt{2 / \beta}$ and $k^{\prime}=$ $(9 / 4)\left\{\sqrt{2 / \beta}+(2 / \beta\}\right.$. Also, $\beta$ is equal to $\omega r^{2} / v$ and $r$ is the radius of the sphere. Note that $M_{d}, k$ and $k^{\prime}$ are known as the displaced mass, the added mass coefficient, and the damping coefficient, respectively. Assuming that the volume of the sphere is equal to a cube whose side length is equal to $d$, the effective radius of the sphere can be approximated as

$$
r_{\mathrm{eff}}=d \cdot\left(\frac{3}{4 \pi}\right)^{1 / 3} .
$$

Substituting the effective radius $r_{\text {eff }}$ for the radius $r$ in (18), we obtain the beam's total effective drag force due to the end effect.

Using (17) and (18), $F_{d}$ and $F_{\mathrm{se}}$ can be evaluated. Fig. 8(a) shows the frequency response of $F_{d}$. The frequency response of $F_{\mathrm{se}}$ is exactly the same as the Q3D-Stokes curve shown in Fig. 7(b). The ratio of $F_{d}$ and $F_{\text {se }}$ (i.e., the $F_{d} / F_{\text {se }}$ ratio), which can be considered as the error due to neglecting the end effect of a single finger, is shown in Fig. 8(b). The figure also shows the ratios of ratio of $F_{d}$ and $F_{s}$ (no edge effect, (16) is used for $F_{s}$ ). Obviously, increasing the ratio of finger length $(l)$ and 


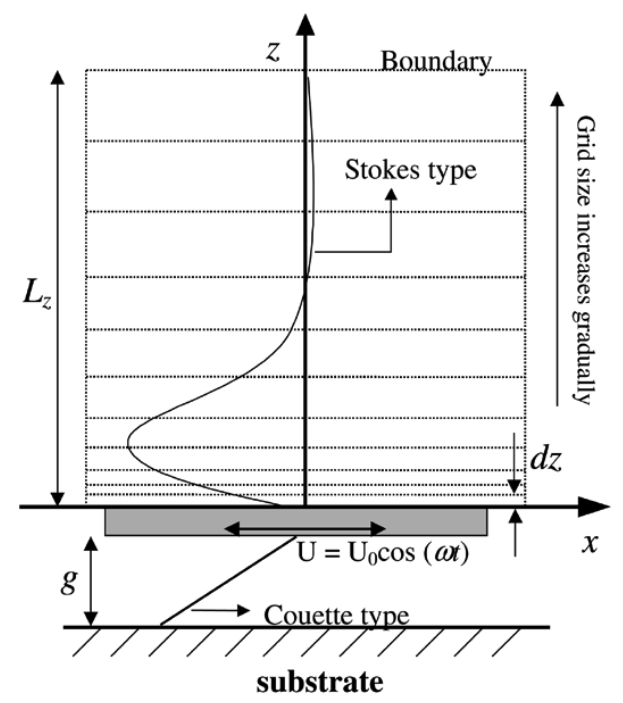

(a)

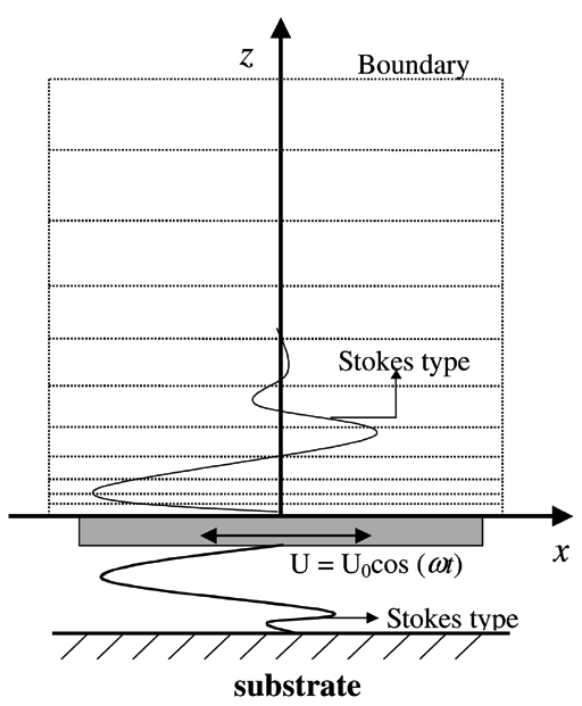

(b)

Fig. 12. Velocity profiles below and above a laterally oscillating plate. (a) At low frequency. (b) At high frequency.

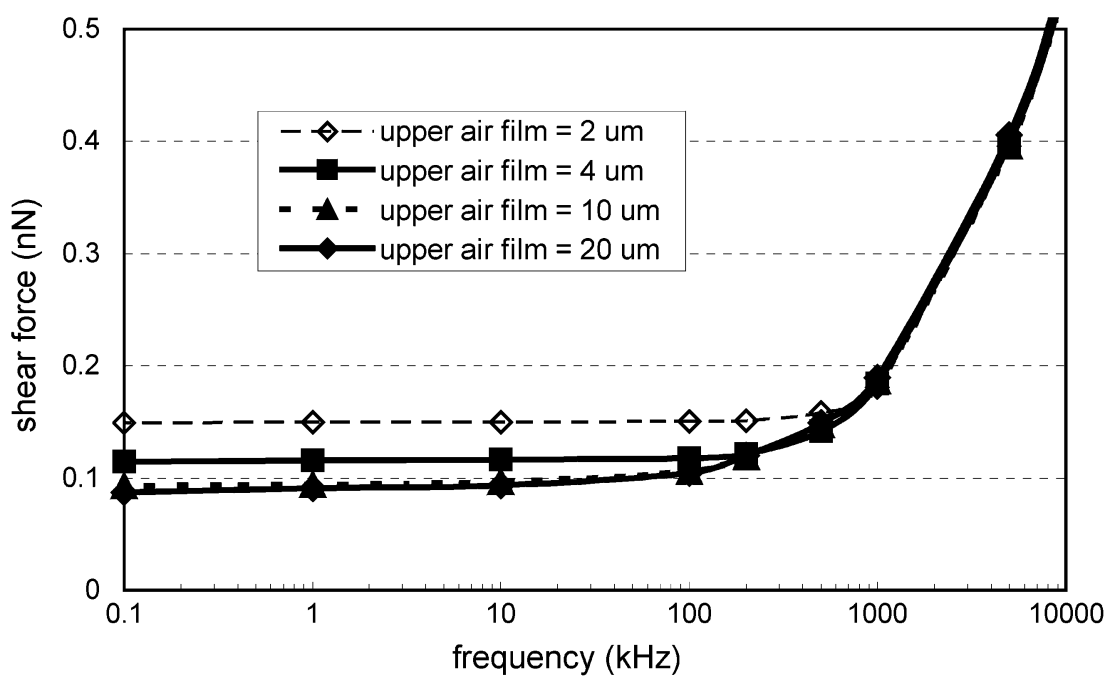

Fig. 13. The frequency responses of the shear force simulated by the FDM solver with different upper air film thickness $L_{Z}$.

width $(d)$ will reduce the $F_{d} / F_{\mathrm{se}}$ ratio (or the $F_{d} / F_{s}$ ratio). This indicates that the 1-D models as well as the Q3D-Stokes model will provide more accurate result for longer finger because the error due to ignoring the end effect can be minimized. Also, the end effect can be further minimized if the edge effect is considered. This is one of the advantages of the Q3D-Stokes model over the 1-D analytical approaches.

\section{B. Comparison of Simulated Results by the FDM Q3D-Stokes Solver and the Macromodels}

As described in Section III, in order to efficiently calculate the damping effect with little compromise in numerical accuracy, the Arnoldi algorithm can be applied to generate macromodels from the system matrices formulated by the FDM Q3D-Stokes solver. Fig. 6 shows that the transient result simulated by the macromodel of order 10 agrees with that by the FDM solver very well. Fig. 6(b) shows the relative errors of total damping forces between different orders of the macromodels and the
FDM solver. The figure indicates that the steady-state error between each macromodel and the FDM Q3D-Stokes solver converges to about $0.1 \%$, while the transient error (before $0.01 \mathrm{~ms}$ ) are heavily depends on the orders of macromodels. The discrepancy between the second-order macromodel result and the FDM result is about $4 \%$, but the tenth-order macromodel result matches with the FDM result well (within 1\%). The error decreases as the order of the macromodel increases.

As aforementioned, the macromodel is in fact a truncated series of the original system. Our results show that the first 10 or the first 20 terms of the series (macromodels with order 10 or 20) can reasonably approximate the original system. This indicates that the first 10 or 20 terms are the most representative terms of the series for the system. In other words, the contribution of each remaining term of the series is relatively insignificant. In fact, our simulations show that this unsteady error between the full-FDM model and the macromodel indeed decreases as the order increases. However, if the orders of the models are greater than 20, the reduction of the unsteady error becomes 


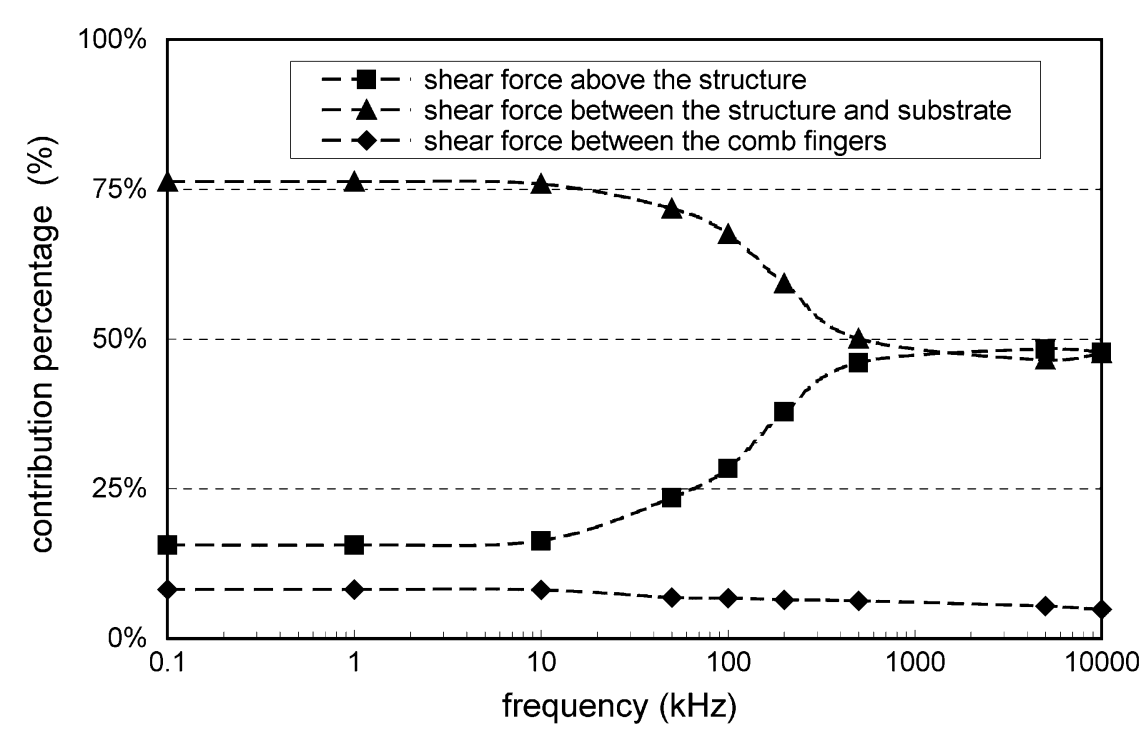

Fig. 14. The contributions of shear force versus frequency for different parts of the comb drive device.

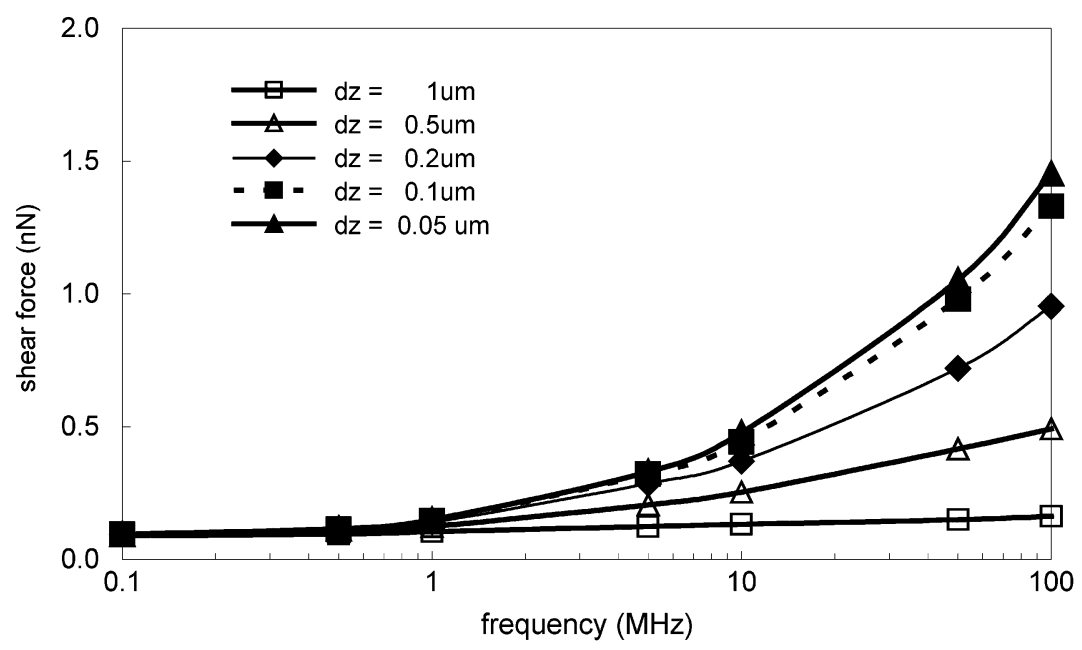

Fig. 15. The relationship between the frequencies and the shear forces for different minimum grid size $d z$. The accuracy at higher frequency $(>1 \mathrm{MHz})$ is strongly dependent on the minimum grid size $d z$.

slow with the increase of the order. Since the order of the original full-FDM model is as high as 84442 , it is not surprised that much more terms of the series are required to make a significant reduction in the unsteady error. The highest order of macromodel we have generated is about 100 . This initial unsteady error is less than but still close to $0.5 \%$.

Table II lists the computational costs as well as the speed-up factors of the macromodels for this simulated case. The required computational costs for generating macromodels of different orders (i.e., the generation time) are also indicated in the table. Note that the generation time is a "one-time charge" during this macromodeling process. Once a macromodel is generated, all the future simulations using the macromodel will not incur the generation time again. Compared with the FDM solver, the speedup of the macromodel with order 20 is about 600 , and the speed-ups are as high as over 5000 for lower-ordered macromodels. Note that all the simulations are performed using a Linux-based PC with single Pentium $42.8 \mathrm{GHz}$ CPU and $1 \mathrm{~GB}$ RAM.

\section{System-Level Simulation and Comparison With Measured Results}

The macromodels generated by the Arnoldi algorithm can be readily inserted into system-level simulators, such as SPICE, Saber, or Simulink, for transient and frequency-response analysis. Fig. 9 is a Simulink system block diagram, which shows that a lateral air damping macromodel is inserted into a secondorder dynamic system model. Table III lists the dimensions of six different devices measured and simulated in this paper. The effective masses and the effective spring constants, which will be required for system-level dynamical analyses, are calculated using the analytical formula in [5]. Note that the effective spring constants are properly adjusted so that the simulated resonance frequencies match with the measured data [3], [11].

Fig. 10 shows the simulated and experimental frequency response of the comb drive device with a folded-beam length of $228 \mu \mathrm{m}$. The simulated result is obtained using a twentieth-order macromodel. Fig. 11 shows the simulated and measured combdrive quality factors versus different folded-beam lengths (see 

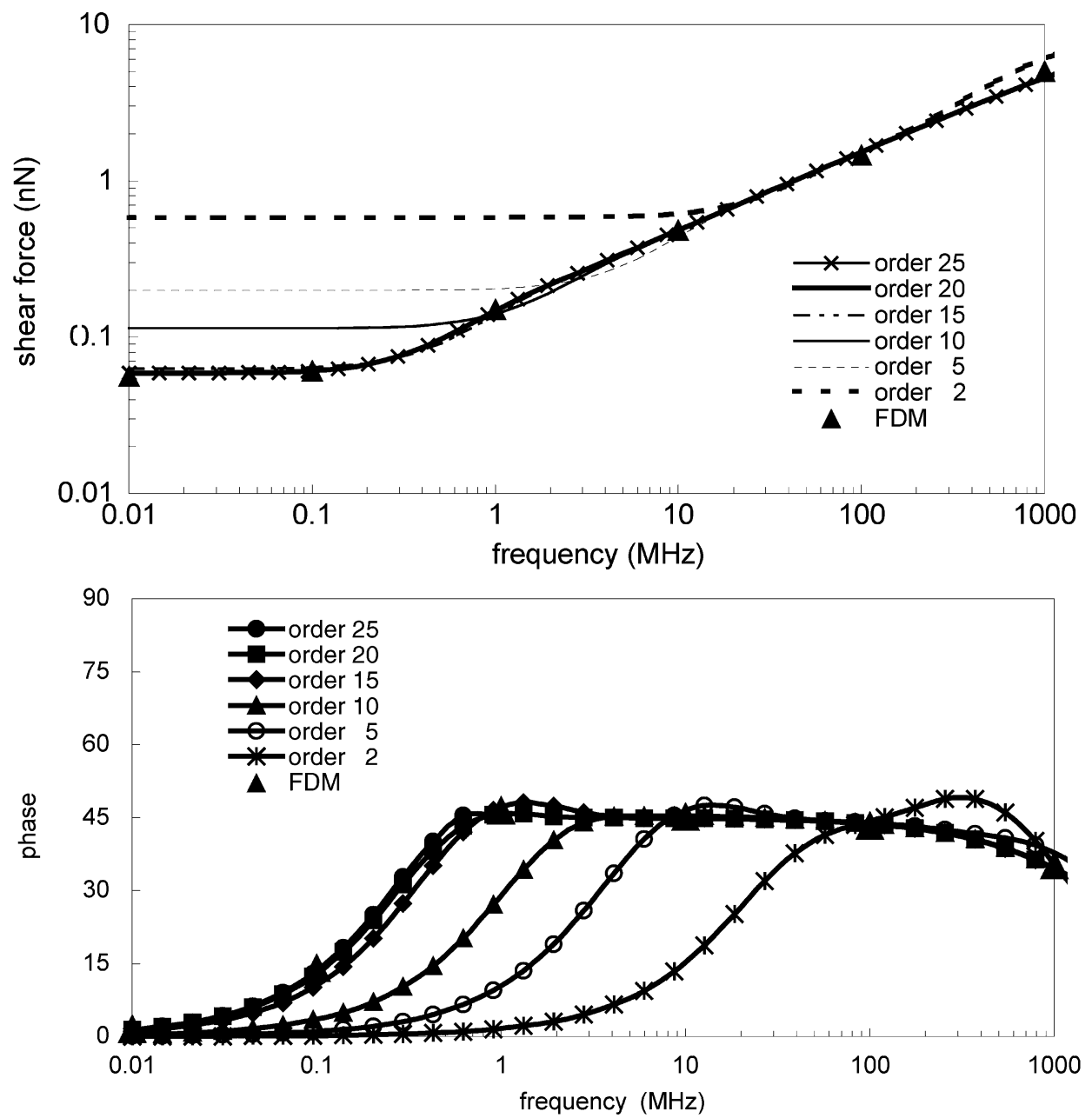

Fig. 16. The frequency responses of macromodels with different orders.

TABLE IV

THE SUITABLE OPERATING FreQuenCy RANGES FOR VARIOUS COMBINATIONS OF $L_{Z}$ 'S AND $d z$ S. THE Numbers INSIDE THE PARENTHESES ARE THE NODE NUMBERS USED FOR THESE CASES

\begin{tabular}{|c|c|c|c|c|c|}
\hline & \multicolumn{4}{|c|}{ Minimum grid size $(d z)$} \\
\hline & & $0.5 \mu \mathrm{m}$ & $0.2 \mu \mathrm{m}$ & $0.1 \mu \mathrm{m}$ & $0.05 \mu \mathrm{m}$ \\
\hline \multirow{4}{*}{ 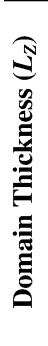 } & $\stackrel{g}{\mathrm{~N}}$ & $\begin{array}{c}\mathrm{N} / / \mathrm{A} \\
(57785)\end{array}$ & $\begin{array}{c}\mathrm{N} / / \mathrm{A} \\
(137795) \\
\end{array}$ & $\begin{array}{c}\text { N/A } \\
(271145) \\
\end{array}$ & $\begin{array}{c}1 \mathrm{M} \sim 10 \mathrm{M}(\mathrm{Hz}) \\
(537845)\end{array}$ \\
\hline & $\stackrel{\xi}{\xi}$ & $\begin{array}{c}\mathrm{N} / / \mathrm{A} \\
(75565)\end{array}$ & $\begin{array}{l}200 \mathrm{k} \sim 500 \mathrm{k}(\mathrm{Hz}) \\
\quad(182245)\end{array}$ & $\begin{array}{c}200 \mathrm{k} \sim 5 \mathrm{M}(\mathrm{Hz}) \\
(360045)\end{array}$ & $\begin{array}{c}200 \mathrm{k} \sim 10 \mathrm{M}(\mathrm{Hz}) \\
\quad(715645)\end{array}$ \\
\hline & $\stackrel{\Xi}{\Xi}$ & $\begin{array}{c}100 \sim 100 \mathrm{k}(\mathrm{Hz}) \\
(128905)\end{array}$ & $\begin{array}{c}100 \sim 500 \mathrm{k}(\mathrm{Hz}) \\
(315595)\end{array}$ & $\begin{array}{c}100 \sim 5 \mathrm{M}(\mathrm{Hz}) \\
(626745)\end{array}$ & $\begin{array}{c}100 \sim 10 \mathrm{M}(\mathrm{Hz}) \\
(1249045)\end{array}$ \\
\hline & 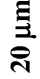 & $\begin{array}{c}10 \sim 100 \mathrm{k}(\mathrm{Hz}) \\
(217805)\end{array}$ & $\begin{array}{c}10 \sim 500 \mathrm{k}(\mathrm{Hz}) \\
(537845)\end{array}$ & $\begin{array}{l}10 \sim 5 \mathrm{M}(\mathrm{Hz}) \\
(1071245)\end{array}$ & $\begin{array}{l}10 \sim 10 \mathrm{M}(\mathrm{Hz}) \\
(2138045)\end{array}$ \\
\hline
\end{tabular}

Table III) [12]. The comb devices are driven electrostatically. The suspended comb shuttle and one of the fixed combs are grounded. The other fixed comb is biased at $70 \mathrm{~V}$ imposed with a sinusoid of $20 \mathrm{~V}$ (peak-to-peak). The amplitude of structure oscillation is visually measured using an optical microscope with a CCD camera (SONY SSC-DC54A). The images of oscillating structures are analyzed using the NI Vision Assistant to estimate the maximum amplitude of oscillation. Note that this measurement method is straightforward but has relatively low resolution, which is reflected in a larger error bars for those measured points in Figs. 10 and 11.

As shown in Fig. 11, all the 1-D analytical approaches [2], [4], [8] overpredicts the quality factors by about $10 \%-30 \%$ because these approaches neglect both the end effect and the edge effect. The results by the Q3D-Stokes macromodel match the measured results very well (less than $10 \%$ discrepancy). It has to be stressed that the good agreement between the Q3D-Stokes macromodel and the measured data is probably due to the cancellation of the errors by neglecting the end effect and neglecting the gas rarefaction effect. The quality factor of the device decreases if the gas rarefaction is not considered, while the quality factor increases if the end effect is ignored.

\section{DISCUSSION}

In previous sections, we have demonstrated that the proposed methodology for lateral-damping macromodeling can be easily implemented, as shown in Fig. 2. In this section, we will address several important issues for appropriately applying this methodology on MEMS devices. In terms of physical consideration, the velocity profiles are heavily dependent on the oscillation frequency, and therefore creating suitable computational domain 


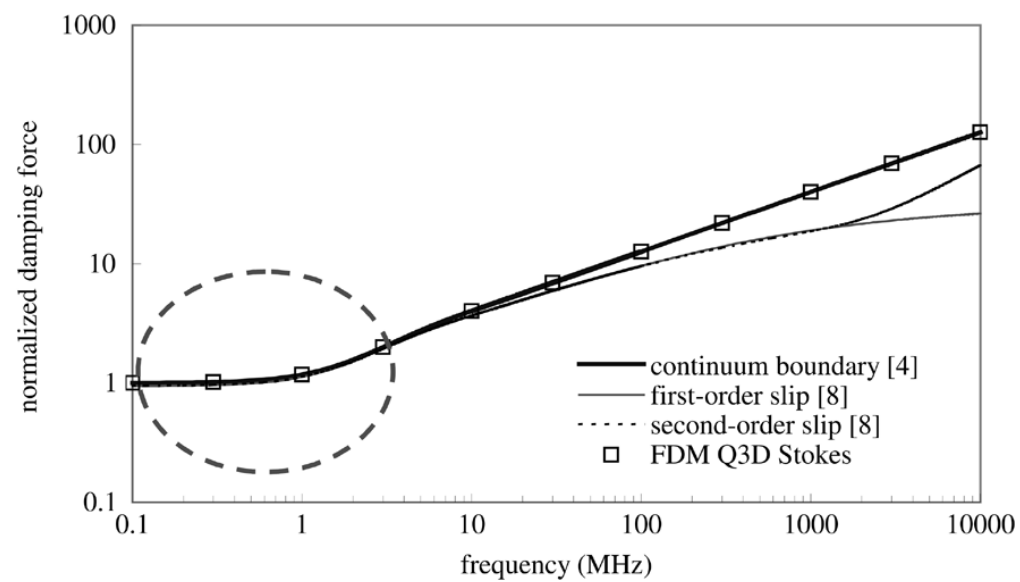

(a)

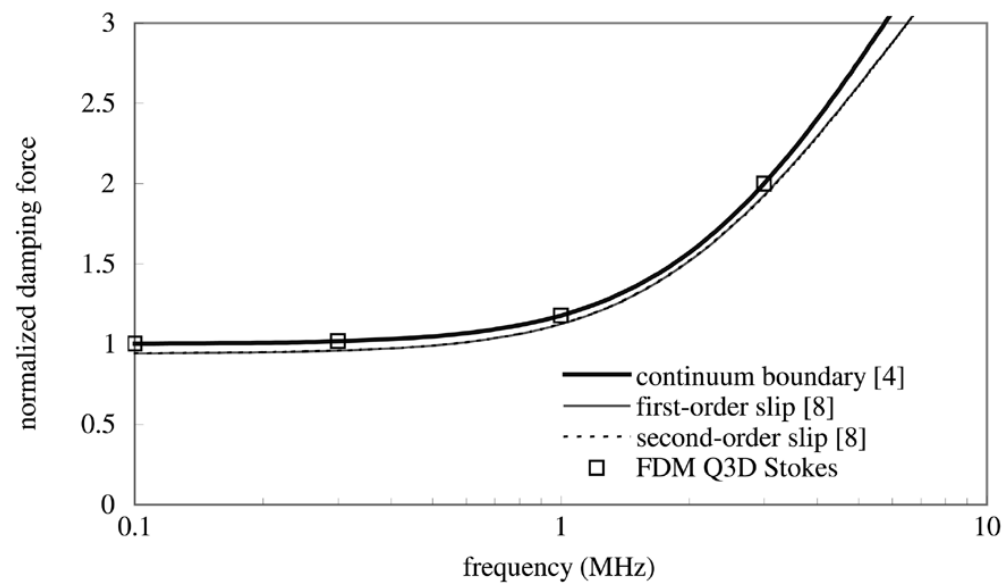

(b)

Fig. 17. (a) The comparison between the results by the FDM Q3D-Stokes equation and by various 1-D analytical solutions with or without slip boundary conditions. (b) The callout of the circled region in (a).

is critical to accurately capture the velocity profiles. Also, in terms of numerical consideration, each generated macromodel can be considered as a truncated Taylor's series of the original system transfer function, and therefore choosing sufficient order for macromodels is important to accurately approximate the original system.

\section{A. Considerations for Computational Domains}

Here, we will discuss the discretization issues of creating appropriate meshes for the FDM Q3D-Stokes solver. It has to be emphasized that the discussions here are generic, so they are also useful for employing commercial FEM CFD packages to model lateral damping.

1) Computational Boundary Depth: Fig. 12(a) shows that when a structure oscillates at relatively low frequency, the air film above the structure is of Stokes-type, and the air film underneath the structure is of Couette-type. As the frequency decreases, the penetration depth of the velocity profile above the plate increases, and, therefore, a larger thickness $L_{Z}$ (the upper air-film thickness) for the computational domain is required [see Fig. 12(a)]. Otherwise, the zero-velocity condition on the top boundary of the air film cannot be correctly specified.
Fig. 13 shows the relationship between $L_{Z}$ and the simulated shear force of the device. When the frequency is higher than $1 \mathrm{MHz}$, the shear forces calculated by the models with different $L_{Z}$ are the same. However, as the frequency decreases, the models with smaller $L_{Z}$ overestimate the damping. Fig. 13 also indicates that as the frequency is less than $100 \mathrm{kHz}$, the total shear force for the case with $L_{Z}=10 \mu \mathrm{m}$ converges with the results for the case with $L_{Z}=20 \mu \mathrm{m}$ (less than 3\% discrepancy), which indicates that the minimum required $L_{Z}$ is about $10 \mu \mathrm{m}$ in order to correctly model the velocity profile for the upper air film.

Fig. 14 shows the damping forces contributed by different parts of the air films surrounding the comb-drive device versus frequency. For this case, $L_{Z}$ is equal to $10 \mu \mathrm{m}$. The damping force contributed by the air film above the movable structure is comparable to the damping contributed by the air film between the movable structure and the substrate when the frequency is higher than $500 \mathrm{kHz}$. The contribution of the damping by the air film between the movable structure and the substrate decreases as the oscillation frequency decreases. This phenomenon is due to the fact that in low frequency range, the lateral damping contributed by the air film above the plate, which is the Stokes-type flow, is much less than that contributed by the air film between the plate and the substrate, which is the Couette-type flow [2] 
TABLE V

The Advantages ANd Limitation BetweEn DifFERENT APPROACHES OF MODELing LATERAL AiR DAMPING EFFECTS

\begin{tabular}{c|c|c|c|c}
\hline & $\begin{array}{c}\text { 1-D Anal. } \\
\text { Models }[2,4,8]\end{array}$ & $\begin{array}{c}\text { BEM Solvers } \\
{[9,10,11]}\end{array}$ & $\begin{array}{c}\text { FDM/FEM } \\
\text { Solvers [17] }\end{array}$ & $\begin{array}{c}\text { Macromodel } \\
\text { in this Work }\end{array}$ \\
\hline Computation Cost & Excellent & Fair & Poor & Excellent \\
\hline Model Creation Cost & N/A & Good & Fair & Fair \\
\hline Time-domain Analysis & Yes & No & Yes & Yes \\
\hline 3D (edge effect) & No & Yes & Yes & Yes \\
\hline System-level simulations & Yes & No & No & Yes \\
\hline Overall Accuracy & Poor & Excellent & Excellent & Good \\
\hline Parameterization & Yes & No & No & No \\
\hline Slip-flow effect & Yes ([8] only) & Yes & N/A & No \\
\hline
\end{tabular}

(see Fig. 12(a)). This also implies that at low frequency operation, an insufficient $L_{Z}$ will not introduce too much error because damping effect contributed by the top air film becomes insignificant. For our simulated cases, the gap between the fingers and the substrate is $2 \mu \mathrm{m}$. Therefore, from Figs. 13 and 14, we may conclude that as long as $L_{Z}$ is at least $10 \mu \mathrm{m}$ or longer (i.e., at least five times greater than the minimum gap between the movable structure and the fixed structure), the overall error caused by an insufficient $L_{Z}$ is less than $3 \%$ as the frequency is around $100 \mathrm{~Hz}$.

2) Required Minimum Mesh Size at High Frequency: As the oscillating frequency increases, the Stokes-type velocity profiles will extend only to a short distance from the structure surface [as shown in Fig. 12(b)], and a finer discretization in the $z$ direction is required on the regions close to the top or the bottom surfaces of the plate. Therefore, on the top surface of the movable structure (i.e., adjacent to the surface of $z=0$ in Fig. 12), a minimum grid size (in $z$ direction), indicated by $d z$, is used. Then the grid size (in $z$ direction) increases gradually toward the top boundary of the computational domain, as shown in Fig. 12. For the regions between the movable plate and the substrate, the grid size is fixed at $d z$. Fig. 15 shows the relationship between the frequencies and the shear forces for different minimum grid size $d z$. Obviously, at higher frequency range (i.e., greater than $200 \mathrm{kHz}$ ), only the results with smaller $d z$ s will converge with a discrepancy of $2 \%$ up to $5 \mathrm{MHz}$ (i.e., $d z=0.1 \mu \mathrm{m}$ and $d z=0.05 \mu \mathrm{m})$. This phenomenon verifies that finer meshes are required to accurately resolve more "packed" velocity profiles. Since most comb-drive-type devices are operated at frequencies less than $500 \mathrm{kHz}$, it is reasonable to conclude that 0.1 $\mu \mathrm{m}$ is an appropriate value of $d z$ for most cases.

\section{B. Choosing Appropriate Orders of Macromodels}

As described in previous section, the Arnoldi algorithm can be considered as a variant of order reduction technique using the truncated Taylor's series. Therefore, macromodels with insufficient orders will certainly result in inaccurate approximation of the original system behaviors. Here, we will present the study of the required orders of macromodels under different operating frequencies. Fig. 16 shows the frequency responses of the total lateral damping forces for macromodels with different orders.
The results by the FDM Q3D-Stokes solver are also shown in the figure. Since the FDM model is too large to be analyzed in frequency domain using typical eigendecomposition techniques, its frequency response is in fact extracted from the transient results with different sinusoidal-excitation frequencies. When the order is greater than 20, the discrepancy between the FDM solver and the macromodel is less than $1 \%$ for a wide range of operating frequency (from dc to $1 \mathrm{GHz}$ ). Therefore, for the specific comb-drive structure studied in this paper, we can conclude that the macromodels with orders greater than 20 are required for less than $1 \%$ error. Note that this $1 \%$ discrepancy is due to order reduction, and is independent of the error due the simplification of the governing equation.

\section{Neglecting Gas Rarefaction Effect}

As described in Section II, the gas rarefaction effect is not considered in the Q3D-Stokes equation. Here, we perform simulations of a testing structure to study the modeling error caused by neglecting the gas rarefaction effect. The testing structure is a flat plate over a fixed substrate. The plate dimensions are 300 $\mu \mathrm{m}$ in length, $300 \mu \mathrm{m}$ in width and $2 \mu \mathrm{m}$ in thickness. The gap between the plate and the substrate is $2 \mu \mathrm{m}$. The computational domain is the air film between the plate and the substrate. The total number of the nodes for the FDM Q3D-Stokes solver is 79 331. The mean free path of the air is $64 \mathrm{~nm}$. The plate oscillates transversely above the substrate. Fig. 17 shows the results by the FDM Q3D-Stokes solver and by various 1-D analytical models with or without slip boundary conditions [4], [8]. The damping forces in the figure are normalized by the result of the 1-D analytical solution (continuum boundary condition) at $10 \mathrm{~Hz}$. Note that the FDM-Q3D-Stokes result is the damping force on the middle of the bottom surface of the oscillating plate, so that it can be used to compare with the results by the 1-D analytical solutions appropriately (i.e., semiinfinite-plate assumption). It is reasonable that the FDM-Q3D-Stokes result is the same as that of the 1-D solution with continuum boundary. However, in the frequency range around $100 \mathrm{~Hz}-1 \mathrm{MHz}$, there is a $6 \%$ (or less) discrepancy between the result by the FDM-Q3D-Stokes solver and the results by the 1-D models with slip boundary conditions. The discrepancy exceeds $10 \%$ as the frequency is greater than 13.5 MHz, and exceeds $20 \%$ as the frequency is above $64 \mathrm{MHz}$. 


\section{Guidelines of Macromodeling}

We summarize the discussions of previous sections and propose the guidelines of using this macromodeling approach. The suitable operating frequency ranges for converged results are listed in Table IV for different $d z \mathrm{~s}$ and $L_{Z}$ s. The numbers inside the parentheses are the node numbers used for these cases. Note that although these results are obtained by simulating the comb structure shown in Fig. 1, we believe they are also applicable for other similar MEMS devices in translational lateral (in-plane) motions. The guidelines are summarized as follows.

1) Finer discretization is required on the region close to the surfaces of the movable structure in order to capture more spatially packed velocity profiles at high frequency range. The suggested $d z$ is 0.1 and $0.05 \mu \mathrm{m}$ for the maximum operation frequencies of 5 and $10 \mathrm{MHz}$, respectively.

2) Consideration of the depth of computational domain is required to correctly model the velocity profiles with longer penetration depths at low frequencies. The suggested $L_{Z}$ is at least five times greater than the minimumgapbetween the movable structure and the fixed structure.

3) It is essential to choose appropriate orders of macromodels for any model-order reduction processes. For this case, orders greater than 20 will be adequate for a wide range of frequency. Devices with more complicated geometries may require higher orders. Fortunately, it is quite straightforward to estimate the required orders because usually the cost of generating macromodels is insignificant.

\section{CONCLUSION}

This paper presents a lateral air damping macromodeling methodology that employs the Q3D Stokes equation and the Arnoldi-based MOR technique. The derivation of the governing equation and the theory of the Arnoldi-based technique are described. The comparison of errors and speedup factors for macromodels with different orders are also demonstrated. The generated macromodels are successfully inserted into the Simulink for system level analysis, and the results are also verified with the experimental data. The macromodels are more than 600 times faster than the FDM Q3D-Stokes solvers. The discrepancy of the simulated and measured quality factors are less than $10 \%$, which is a significant improvement compared with the results by the widely used 1-D analytical approaches. Table V shows the comparison of the advantages and limitations between different approaches for modeling lateral air damping effects. The table indicates that the macromodels not only are as efficient as the 1-D analytical approaches, but also are capable of providing reasonably accurate results for the devices with very complicated geometries. Also, the generated macromodels are readily for system-level transient or frequency analyses which cannot be achieved by the BEM approaches, and require much less computational cost than the full 3-D FEM Stokes solvers.

\section{APPENDIX I}

Since detailed derivation for a 3-D case is very tedious, here we employ a 1-D case to demonstrate how A, B, C, and D matrices are created by the FDM formulation. Fig. 3 shows a schematic of a 1-D case. The top surface of a plate (the dotted line) is moving with a velocity $U_{0}$ in the $x$ direction. The velocity profile is represented as the curved line in the figure. The coordinates of the Nodes $i-1, i$ and $i+1$ are $z_{i-1}, z_{i}$, and $z_{i+1}$, respectively. The corresponding velocities at each node are $u_{i-1}, u_{i}$ and $u_{i+1}$. The 1 -D governing equation can be written as

$$
\frac{\partial u}{\partial t}=v \nabla^{2} u=v\left(\frac{\partial^{2} u}{\partial z^{2}}\right)
$$

The finite-difference equation for Node $i$ can be written as

$$
\begin{aligned}
\frac{\partial u_{i}}{\partial t}= & v\left[\frac{\left(\frac{u_{i+1}-u_{i}}{z_{i+1}-z_{i}}\right)-\left(\frac{u_{i}-u_{i-1}}{z_{i}-z_{i-1}}\right)}{\left(\frac{z_{i+1}-z_{i-1}}{2}\right)}\right] \\
= & \frac{2 v}{\left(z_{i+1}-z_{i-1}\right)}\left[\frac{1}{\left(z_{i+1}-z_{i}\right)} \cdot u_{i+1}+\frac{1}{\left(z_{i}-z_{i-1}\right)}\right. \\
& \left.\cdot u_{i-1}+\frac{\left(z_{i-1}-z_{i+1}\right)}{\left(z_{i+1}-z_{i}\right) \cdot\left(z_{i}-z_{i-1}\right)} \cdot u_{i}\right] \cdot
\end{aligned}
$$

Note that $u_{i}$ and $u_{i+1}$ are the unknown velocity variables to be solved. In this case, $u_{i-1}$ is the given boundary condition, and is equal to $U_{0}$. After combining the finite difference equations for all the nodes ( $N$ nodes in total) in the computational domain, a set of dynamic system equations (6) can be formed as [see (7)]

$$
\dot{\vec{u}}=\mathbf{A} \cdot \vec{u}+\mathbf{B} \cdot v_{\text {in }}
$$

where $\vec{u}=\left[\begin{array}{llll}u_{1} & u_{2} & \cdots & u_{N}\end{array}\right]^{T}, \mathbf{A}$ contains the coefficients corresponding to nodes with unknown velocities, $v_{\text {in }}$ is equal to $U_{0}$ in this case, and $\mathbf{B}$ contain the coefficients corresponding nodes on the prescribed boundary.

The shear stress on the moving surface (Node $i-1$ ) due to the velocity profile is

$$
\tau=-\left.\mu \frac{\partial u(z)}{\partial z}\right|_{z=z_{i-1}} .
$$

The velocity profile can be approximated by a second-order polynomial of $z$

$$
u(z)=c_{1} z^{2}+c_{2} z+c_{3}
$$

where $c_{1}, c_{2}$, and $c_{3}$ are unknown coefficients. After the velocity profile is solved, we substitute $u\left(z_{i-1}\right)=U_{0}, u\left(z_{i}\right)=u_{i}$, and $u\left(z_{i+1}\right)=u_{i+1}$ into (A2), and obtain three linear equations. Thus the known coefficients $c_{1}, c_{2}$, and $c_{3}$ can be evaluated. In order to reduce the complexity of representing the shear stress on Node $i-1$, we make a coordinate transformation so that $z_{i-1}$ is equal to 0 . Therefore, the shear stress on Node $i-1$ is

$$
\begin{aligned}
\tau= & -\left.\mu \frac{\partial u(z)}{\partial z}\right|_{z=0}=-\left.\mu\left(2 c_{1} z+c_{2}\right)\right|_{z=0}=-\mu \cdot c_{2} \\
= & -\mu\left(\frac{z_{i+1}}{z_{i}\left(z_{i+1}-z_{i}\right)} \cdot u_{i}-\frac{z_{i}}{z_{i+1}\left(z_{i+1}-z_{i}\right)}\right. \\
& \left.\cdot u_{i+1}-\frac{z_{i+1}+z_{i}}{z_{i} z_{i+1}} \cdot U_{0}\right) .
\end{aligned}
$$


Obviously, if the output of the system is the shear stress (i.e., the output $\vec{y}$ of (7) is equal to $\tau$ ), we may write

$$
\vec{y}=\mathbf{C}^{T} \cdot \vec{u}+\mathbf{D} \cdot v_{\text {in }}
$$

where $\mathbf{C}$ contains the coefficients corresponding to nodes with unknown velocities, and $\mathbf{D}$ contains the coefficients corresponding nodes on the prescribed boundary condition. Note that $v_{\text {in }}$ is also equal to $U_{0}$ in this case. In this 1-D case, the output vector $\vec{y}$ is in fact a scalar variable. For 2- or 3-D cases, the output vector $\vec{y}$ contains the shear stress distribution on the surface of the moving plate.

\section{APPENDIX II}

The system matrices of a comb-drive macromodel with order 5 is listed

$$
\begin{aligned}
\mathbf{A}_{q} & =10^{7} \times\left[\begin{array}{lllll}
-2.85 & -2.19 & -1.87 & -1.79 & -1.25 \\
-2.19 & -2.18 & -1.86 & -1.78 & -1.24 \\
-1.87 & -1.86 & -2.79 & -2.68 & -1.86 \\
-1.79 & -1.78 & -2.68 & -4.86 & -3.38 \\
-1.25 & -1.24 & -1.86 & -3.38 & -3.37
\end{array}\right], \\
\mathbf{B}_{q} & =10^{9} \times\left[\begin{array}{l}
1.79 \\
1.37 \\
1.17 \\
1.12 \\
0.78
\end{array}\right] \\
\mathbf{C}_{q} & =-10^{-10} \times\left[\begin{array}{lllll}
0.564 & 0.378 & 0.356 & 0.436 & 0.439
\end{array}\right]^{T} \\
\mathbf{D}_{q} & =3.73 \times 10^{-9} .
\end{aligned}
$$

\section{ACKNOWLEDGMENT}

The authors would like to thank Prof. S. D. Senturia of the Massachusetts Institute of Technology, Cambridge, and Dr. T. Korsmeyer of Coventor Inc. for comments and suggestions on frequency and mesh convergence study.

\section{REFERENCES}

[1] S. D. Senturia, Microsystem Design. Boston, MA: Kluwer, 2001.

[2] Y.-H. Cho, A. P. Pisano, and R. T. Howe, "Viscous damping model for laterally oscillating microstructures," J. Microelectromech. Syst., vol. 3, no. 2, pp. 81-87, Jun. 1994.

[3] Y.-J. Yang, M. Gretillat, and S. D. Senturia, "Effect of air damping on the dynamics of nonuniform deformations of microstructures," in Proc. 9th Int. Conf. Solid-State Sensors and Actuators (Transducers'97), Chicago, IL, Jun. 1997, pp. 1093-1096.

[4] X. Zhang and W. C. Tang, "Viscous air damping in laterally driven microresonators," Sens. Materials, vol. 7, no. 6, pp. 415-430, 1995.

[5] W. C. Tang, T. H. Nguyen, and R. T. Howe, "Laterally driven polysilicon resonant microstructures," in Tech. Dig. IEEE Micro Electromechanical Systems Workshop, Salt Lake City, UT, Feb. 20-22, 1989, pp. 53-59.

[6] K. B. Lee and Y. H. Cho, "Laterally driven electrostatic repulsive-force microactuators using asymmetric field distribution," J. Microelectromech. Syst., vol. 10, no. 1, pp. 128-136, Mar. 2001.

[7] G. K. Fedder and T. Mukherjee, "Physical design for surface-micromachined MEMS," in Proc. 5th ACM/SIGDA Physical Design Workshop, Reston, VA, Apr. 15-17, 1996, pp. 53-60.

[8] T. Veijola and M. Turowski, "Compact damping models for laterally moving microstructures with gas rarefaction effects," J. Microelectromech. Syst., vol. 10, no. 2, pp. 263-273, Jun. 2001.
[9] N. R. Aluru and J. White, "A fast integral equation technique for analysis of microflow sensors based on drag force calculations," in Proc. MSM, Santa Clara, CA, Apr. 1998, pp. 283-286.

[10] X. Wang, M. Judy, and J. White, "Validating fast simulation of air damping in micromachined devices," in Proc. IEEE 15th Int. Conf. Micro Electro-Mechanical Systems Workshop (MEMS 2001), Las Vegas, NV, Jan. 2002, pp. 210-213.

[11] W. Ye, X. Wang, W. Hemmert, D. Freeman, and J. White, "Air damping in laterally oscillating microresonators: A numerical and experimental study," J. Microelectromech. Syst., vol. 12, no. 5, pp. 557-566, Oct. 2003.

[12] P.-C. Yen and Y.-J. Yang, "Macromodels of 3D lateral viscous damping effects for MEMS devices," in Proc. 12th Int. Conf. Solid-State Sensors and Actuators (Transducers'03), Boston, MA, Jun. 2003, pp. 1848-1851.

[13] Y.-J. Yang, M. Kamon, V. L. Rabinovich, C. Ghaddar, M. Deshpande, K. Greiner, and J. R. Gilbert, "Modeling gas damping and spring phenomena In mems with frequency dependent macromodels," in Proc. IEEE 14th Int. Conf. Micro Electro-Mechanical Systems Workshop (MEMS 2001), Interlaken, Switzerland, Jan. 2001, pp. 365-368.

[14] A. Odabasioglu, M. Celik, and L. T. Pileggi, "PRIMA: Passive reduced-order interconnect macro-modeling algorithm," in Proc. IEEE Trans. Comput.-Aided Des. Integr. Circuits Syst., vol. 17, Aug. 1998, pp. 645-654.

[15] F. Wang and J. White, "Automatic model order reduction of a microdevice using the arnoldi approach," in Proc. 1998 ASME Int. Mechanical Engineering Congress and Exposition (IMECE'98, DSC-Vol. 66), Anaheim, CA, Nov. 1998, pp. 527-530.

[16] T. Bechtold, E. B. Rudnyi, and J. G. Korvink, "Automatic generation of compact electro-thermal models for semiconductor devices," IEICE Trans. Electronics, vol. E86C, no. 3, pp. 459-465, 2003.

[17] CoventorWare2001 Reference Manual: Coventor, Inc., 2001.

[18] MEMS Pro V3.0 Tutorial: MEMSCAP, Inc., 2001.

[19] IntelliSuite Reference Manual: IntelliSense, Inc.

[20] W. H. Press, B. P. Flannery, S. A. Teukolsky, and W. T. Vetterling, Numerical Recipes in C: The Art of Scientific Computing, 2nd ed. Cambridge, U.K.: Cambridge University Press, 1993.

[21] J. Stoer and R. Bulirsch, Introduction to Numerical Analysis, 2nd ed. New York: Springer-Verlag, 1992.

[22] E. B. Rudyni and J. G. Korvink, "Review: Automatic model reduction for transient simulation of MEMS-based devices," Sensors Update, vol. 11, pp. 3-33, 2002.

[23] J. A. Fay, Introduction to Fluid Mechanics. Cambridge: MIT Press, 1994.

[24] MUMPS Design Handbook, MEMSCAP, Inc., 2002.

[25] F. K. Moore, Theory of Laminar Flow. Princeton, NJ: Princeton University Press, 1964.

[26] L. Rosenhead, Laminar Boundary Layers. London, U.K.: Oxford University Press, 1963.

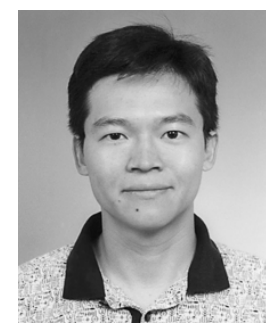

Yao-Joe Joseph Yang (S'98-M'01) received the B.S. degree from the National Taiwan University, Taipei, Taiwan, in 1990, and the M.S. and the $\mathrm{Ph} . \mathrm{D}$. degrees in electrical engineering from the Massachusetts Institute of Technology, Cambridge, in 1997 and 1999, respectively.

From 1999 to 2000, he joined the Coventor Inc., Cambridge, as a senior application engineer. Since 2000, he has been with the Department of Mechanical Engineering, National Taiwan University, Taipei. Currently, he is an Associate Professor. He also serves as the Director for CAD Technology in the Northern NEMS Center sponsored by the National Science Council, Taiwan. Since 2005, he serves as the codirector of the Opto-Electronics Resource Center (OERC) sponsored by the Ministry of Education, Taiwan. His research interests include microelectromechanical systems, nanotechnology, parallel processing, and semiconductor devices and vacuum microelectronics modeling. He has been a consultant for more than three U.S.-based companies and four Taiwan-based organizations. 


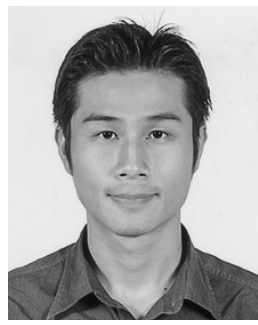

Po-Ching Yen received the B.S. and M.S. degrees in mechanical engineering from National Taiwan University, Taipei, Taiwan, R.O.C., in 2000 and 2002, respectively.

He is now pursuing the $\mathrm{Ph} . \mathrm{D}$. degree in mechanical engineering. From 2001 to 2004, he did research in the area of model order reduction of MEMS devices. Currently his research topic is focus on the control of AFM tips. He has been being involved in several research projects sponsored by the National Science Council, Taiwan. His main research interests include numerical analysis, system dynamics/control, and model order reduction techniques. 